\title{
Panel cointegration testing in the presence of common factors
}

Citation for published version (APA):

Gengenbach, C., Palm, F. C., \& Urbain, J. R. Y. J. (2005). Panel cointegration testing in the presence of common factors. METEOR, Maastricht University School of Business and Economics. METEOR Research Memorandum No. 051 https://doi.org/10.26481/umamet.2005051

Document status and date:

Published: 01/01/2005

DOI:

10.26481/umamet.2005051

Document Version:

Publisher's PDF, also known as Version of record

\section{Please check the document version of this publication:}

- A submitted manuscript is the version of the article upon submission and before peer-review. There can be important differences between the submitted version and the official published version of record.

People interested in the research are advised to contact the author for the final version of the publication, or visit the DOI to the publisher's website.

- The final author version and the galley proof are versions of the publication after peer review.

- The final published version features the final layout of the paper including the volume, issue and page numbers.

Link to publication

\footnotetext{
General rights rights.

- You may freely distribute the URL identifying the publication in the public portal. please follow below link for the End User Agreement:

www.umlib.nl/taverne-license

Take down policy

If you believe that this document breaches copyright please contact us at:

repository@maastrichtuniversity.nl

providing details and we will investigate your claim.
}

Copyright and moral rights for the publications made accessible in the public portal are retained by the authors and/or other copyright owners and it is a condition of accessing publications that users recognise and abide by the legal requirements associated with these

- Users may download and print one copy of any publication from the public portal for the purpose of private study or research.

- You may not further distribute the material or use it for any profit-making activity or commercial gain

If the publication is distributed under the terms of Article $25 \mathrm{fa}$ of the Dutch Copyright Act, indicated by the "Taverne" license above, 


\title{
Panel Cointegration Testing in the Presence of COMMON FACTORS
}

\author{
Christian Gengenbach, Franz C. Palm, Jean-Pierre Urbain* \\ Department of Quantitative Economics, \\ Universiteit Maastricht, The Netherlands \\ November 2005
}

\begin{abstract}
Panel unit root and no-cointegration tests that rely on cross-sectional independence of the panel unit experience severe size distortions when this assumption is violated, as has e.g. been shown by Banerjee, Marcellino and Osbat $(2004,2005)$ via Monte Carlo simulations. Several studies have recently addressed this issue for panel unit root test using a common factor structure to model the cross-sectional dependence, but not much work has been done yet for panel no-cointegration tests.

This paper proposes a model for panel no-cointegration using an unobserved common factor structure, following the work on Bai and $\mathrm{Ng}$ (2004) for panel unit roots. The model enables us to distinguish two important cases: $(i)$ the case when the non-stationarity in the data is driven by a reduced number of common stochastic trends, and (ii) the case where we have common and idiosyncratic stochastic trends present in the data. We study the asymptotic behavior of some existing, residual-based panel no-cointegration, as suggested by Kao (1999) and Pedroni (1999, 2004). Under the DGP used, the test statistics are no longer asymptotically normal, and convergence occurs at rate $T$ rather than $\sqrt{N} T$ as for independent panels. We then examine the properties of residual-based tests for nocointegration applied to defactored data from which the common factors and individual components have been extracted.
\end{abstract}

Keywords: panel cointegration testing, common factors.

\footnotetext{
${ }^{*}$ Corresponding author: Jean-Pierre Urbain, Department of Quantitative Economics Universiteit Maastricht, P.O. Box 616, 6200 MD Maastricht, The Netherlands, j.urbain@ke.unimaas.nl. Previous versions of this paper were presented at the $12^{\text {th }}$ International Panel Conference in Copenhagen, at the Conference on Frontiers in Times Series Analysis in Olbia, at a seminar at the Econometric Institute in Rotterdam and at the NSF/NBER Time Series Conference in Heidelberg. The authors would like to thank conference and seminar participants and in particular Jörg Breitung, Josep Carrion-i-Silvestre, Peter Pedroni and Joakim Westerlund for helpful comments and suggestions on a previous draft. The usual disclaimer applies.
} 


\section{Introduction}

The effect of persistent cross-sectional dependence on panel unit root tests has been recently analyzed and documented in some detail in the literature. As shown by Monte Carlo simulations (Banerjee et al, 2005) or by asymptotic analysis (Lyhagen, 2000; Pedroni and Urbain, 2001), the standard (Levin-Lin-Chu or IPS) panel unit root tests are severely affected in that either they display dramatic size distortions or even worse can be shown to diverge with the cross-sectional dimension of the panel. To overcome these problems, new panel unit root tests have been proposed that model the possibly persistent cross-sectional dependency using a single common factor model (Pesaran, 2004, Philips and Sul, 2003) or $k$-common factor models (Moon and Perron, 2004, Bai and Ng, 2004).

For the case of tests of the null hypothesis of no-cointegration, not much work has been done yet. Banerjee et al. (2004) conduct an extensive Monte Carlo study where they conclude that while all statistics investigated (residual-based tests or likelihood based trace-type test) are affected, the presence of cross-member cointegration appears much less harmful for singleequation tests than for the panel version of the Johansen test. In many cases, the tests are affected by the presence of cointegration between members in such a way that these tests cannot discriminate between cointegration among members and cointegration within, that is for a single member of the panel. Bai and Kao (2004) and Banerjee and Carrion-i-Silvestre (2005) study tests for panel no-cointegration with cross-sectional dependence. Both studies consider residual-based tests for a single cointegration relationship, where the error term of the cointegrating equation follows a common factor structure as in Bai and $\mathrm{Ng}$ (2004). Urbain (2004) on the other hand studies analytically the issue of spurious regression in panels when the units are cointegrated along the cross-sectional dimension, i.e. when there is cross-member cointegration. In contrast to the spurious regression result for independent panel studied by Phillips and Moon (1999), Pedroni (1995) or Kao (1999), in most of the cases considered these estimators are not consistent and actually converge to non-degenerate limiting distribution once the observed non-stationarity is generated by a reduced number of common stochastic trends.

This paper builds on these results and extends the analysis to the analytical study of panel tests for no-cointegration when the cross-sectional dependence in the panel is modelled by a common factor structure following the work of Bai and Ng (2004). Adopting the framework of Bai and $\mathrm{Ng}$ (2004) enables us to consider essentially two different classes of cases that we believe are both of theoretical and empirical relevance: $(i)$ the case where the observed non-stationarity in the variables originates from a reduced number of cross-sectional common trends only; $(i i)$ the case where we have both cross-sectional common stochastic trends as well as idiosyncratic ones. The spurious regression analysis for the former case is reported in Urbain (2004) and corresponds to the cross-member cointegration case. The second case is actually the one considered by Moon and Perron (2004) or Pesaran (2003) in the context of panel unit root analysis and excludes the existence of cross-unit or cross-member cointegration in the panel since both components are $\mathrm{I}(1)$ ).

For both classes of DGP's, we study analytically the behavior of several popular test for panel cointegration including Kao (1999) and Pedroni's (1999, 2004) residual-based panel no-cointegration tests that have been widely used in empirical work in the recent years. For example, when the number of common factors generating the non-stationarity in the panel is kept fixed while the dimension of the panel increases, then the Gaussian limiting results derived for the independent case are not valid anymore. Tests that are based on pooled or 
LSDV estimation of the underlying panel cointegration static regression may in some cases diverge with $\sqrt{N}$ and hence important size distortions are to be expected already in panels with moderate cross-sectional dimension. Group mean statistics are also affected and not asymptotically gaussian anymore.

These results complement and help to have a better understanding of some of the Monte Carlo reported by Banerjee et al. (2004). We then examine the possibilities to test for no-cointegration, by extracting the common factors and individual components from the observed data directly and test for no-cointegration using residual-based panel tests applied to the defactored data.

The remainder of the paper is organized as follows: In Section 2 we present our model for panel no-cointegration with a common factor structure. In Section 3 we examine the asymptotic behavior of some residual-based panel no-cointegration tests when the data is generated by our DGP. Section 4 discusses a possible solution for testing for no-cointegration when the data contains unobserved common factors. In particular, we examine the possibilities of defactoring the observed data before testing, using the methodology from Bai and $\mathrm{Ng}$ (2004). The finite sample behavior of the proposed approach is analyzed in Section 5. In Section 6 we illustrate the approach by revisiting the issue of panel cointegration of the weak PPP hypothesis. Conclusions are drawn in Section 7.

A note on notation: Throughout the text, $M$ is used to denote a generic positive number, not depending on $T$ or $N$. For a matrix $\boldsymbol{A}, \boldsymbol{A}>0$ denotes that $\boldsymbol{A}$ is positive definite. Furthermore, $\|\boldsymbol{A}\|=\operatorname{trace}\left(\boldsymbol{A}^{\prime} \boldsymbol{A}\right)^{\frac{1}{2}}$. We write the integral $\int_{0}^{1} B(r) \mathbf{d} r$ as $\int B$, and $\int_{0}^{1} B(r) B(r)^{\prime} \mathbf{d} r$ as $\int B B^{\prime}$. Furthermore, $\Longrightarrow$ denotes weak convergence, and $\stackrel{p}{\longrightarrow}$ denotes convergence in probability. For any number $x,\lfloor x\rfloor$ denotes the largest integer smaller than $\mathrm{x}$. For any variable $X_{i, t}$, $\tilde{X}_{i, t}=X_{i, t}-\frac{1}{T} \sum_{s=1}^{T} X_{i, s}$. Similarly, for any Brownian motion $B, \tilde{B}=B-\int B$. Throughout the paper we employ sequential limit theory, where we consider $T \rightarrow \infty$ followed by $N \rightarrow \infty$.

\section{The Model}

We consider balanced panels with $N$ cross-sectional units and $T$ time-series observations, indexed by $i=1, \ldots, N$ and $t=1, \ldots, T$ respectively. For each unit in the panel we observe a $(m+1)$-dimensional vector of variables $Z_{i, t}=\left(Y_{i, t}, X_{i, t}^{\prime}\right)^{\prime}$, where $Y_{i, t}$ is a scalar time series and $X_{i, t}$ is a $m$-vector time series. We assume that the DGP for $Z_{i, t}$ has a common factor structure as e.g. in Bai and $\mathrm{Ng}$ (2004), and we assume the presence of $k$ common factors in the data. Furthermore, we assume the number of common factors to be fixed as $T, N \rightarrow \infty$ throughout the paper. Our model is given by

$$
Z_{i, t}=D_{i, t}+\Lambda_{i} F_{t}+E_{i, t}
$$

$t=1, \ldots, T, i=1, \ldots, N . D_{i, t}$ is an unobserved deterministic component such that either $D_{i, t}=0$ for all $i$ and $t$ if there are no deterministic components present, $D_{i, t}=d_{0 i}$ for all $t$ if the data contains individual specific fixed effects, or $D_{i, t}=d_{0 i}+d_{1 i} t$ if the data contains individual specific deterministic components, where the coefficients $d_{0 i}$ and $d_{1 i}$ depend on $i$ only. For the remainder of the paper we assume $D_{i, t}=0$ unless mentioned otherwise. The common component in $Z_{i, t}$ is given by $F_{t}$ in (1). $F_{t}$ is a $k$-vector of common I(1) factors given by

$$
F_{t}=F_{t-1}+f_{t}
$$


where $f_{t}=\Phi(L) \eta_{t}, \Phi(L)=\sum_{j=0}^{\infty} \Phi_{j} L^{j}$. The $(m+1) \times k$ matrix of factor loadings $\Lambda_{i}$ is assumed to be of full rank and block-diagonal, with block-diagonality corresponding to the partition of $Z_{i, t}$, such that

$$
\Lambda_{i}=\left[\begin{array}{cc}
\lambda_{1 i}^{\prime} & 0 \\
0 & \lambda_{2 i}^{\prime}
\end{array}\right] .
$$

As for the vectors of observations $Z_{i, t}$, we have partitions for the unobserved vector of common factors $F_{t}=\left(F_{t}^{Y^{\prime}}, F_{t}^{X^{\prime}}\right)^{\prime}$ where $F_{t}^{Y}$ and $F_{t}^{X}$ have $k_{Y}$ and $k_{X}$ elements respectively, and the partition of $F_{t}$ corresponds to the structure of $\Lambda_{i}$, such that $\lambda_{1 i}$ is $k_{Y} \times 1$ and $\lambda_{2 i}$ is $k_{X} \times m$. The block-diagonal structure for the factor loadings is necessary to ensure that $Y_{i, t}$ and $X_{i, t}$ are not cointegrated when the non-stationarity in the data is driven by the common factors alone. When the idiosyncratic components are non-stationary as well, this assumption on $\Lambda_{i}$ might be relaxed and a more general structure can be considered.

For the idiosyncratic component in (1), $E_{i, t}$, we distinguish two cases, namely stationary and non-stationary idiosyncratic components. For the former case we have

$$
E_{i, t}=e_{i, t}
$$

while in the later case we assume

$$
E_{i, t}=E_{i, t-1}+e_{i, t}
$$

where the stationary vector $e_{i, t}=\Gamma_{i}(L) \varepsilon_{i, t}, \Gamma_{i}(L)=\sum_{j=0}^{\infty} \Gamma_{i j} L^{j}$. Again, we partition $E_{i, t}$ conformable with the data $Z_{i, t}$, such that $E_{i, t}=\left(E_{i, t}^{Y}, E_{i, t}^{X^{\prime}}\right)^{\prime}$, where $E_{i, t}^{Y}$ is a scalar time series and $E_{i, t}^{X}$ has $m$ elements.

For the above given model we specify the following assumptions:

Assumption 1 Common factors: (i) $\eta_{t} \sim$ iid $\left(0, I_{k}\right)$ with finite $4^{\text {th }}$ moments, (ii) $\sum_{j=0}^{\infty} j$. $\left\|\Phi_{j}\right\|<M$, (iii) $\operatorname{rank}(\Phi(1))=k$, (iv) $\mathrm{E}\left\|F_{0}\right\| \leq M$.

Assumption 2 Factor loadings: (i) for non-random $\lambda_{1 i}$ and $\lambda_{2 i},\left\|\lambda_{1 i}\right\| \leq M$ and $\left\|\lambda_{2 i}\right\| \leq M$; for random $\lambda_{1 i}$ and $\lambda_{2 i}$, $\mathrm{E}\left\|\lambda_{1 i}\right\|^{4} \leq M$ and $\mathrm{E}\left\|\lambda_{2 i}\right\|^{4} \leq M$, (ii) $N^{-1} \sum_{i=1}^{N} \Lambda_{i}^{\prime} \Lambda_{i} \stackrel{p}{\longrightarrow} \Sigma_{\Lambda}>0$, (iii) for non-random $\lambda_{1 i}$ and $\lambda_{2 i}, N^{-1} \sum_{i=1}^{N} \lambda_{1 i} \neq 0$ and $N^{-1} \sum_{i=1}^{N} \lambda_{2 i} \neq 0$; for random $\lambda_{1 i}$ and $\lambda_{2 i}, \mathrm{E}\left(\lambda_{1 i}\right) \neq 0$ and $\mathrm{E}\left(\lambda_{2 i}\right) \neq 0$.

Assumption 3 Idiosyncratic components: for each $i=1, \ldots, N$, (i) $\varepsilon_{i, t} \sim$ iid $\left(0, \Sigma_{i}\right)$ with finite $8^{\text {th }}$ moments, and $\varepsilon_{i, t}$ and $\varepsilon_{j, s}$ are independent for any $t, s$ and $i \neq j$, (ii) $\mathrm{E}\left\|\varepsilon_{i, 0}\right\|<M$, (iii) $\Gamma_{i}(L)$ fulfills the random coefficients and summability conditions from Phillips and Moon (1999), Assumptions 1 and 2 on $p .1060$ and p.1061 respectively, (iv) $\operatorname{rank}\left(\Gamma_{i}(1)\right)=m+1$, $\forall i$.

Assumption 4 The errors, $\eta_{t}, \varepsilon_{i, t}$, and the factor loadings $\Lambda_{i}$ are mutually independent groups.

Under the conditions of Assumption 1, the common factors $F_{t}$ are a $k$-dimensional I(1) process and the possibility of cointegration between the common factors is excluded. The full rank assumption on the long-run covariance matrix of $F_{t}$ could in fact be relaxed, as long as the diagonal blocks corresponding to the long-run covariances of $F_{t}^{Y}$ and $F_{t}^{X}$ have at least rank 1 each. The long-run covariance matrix of the common factors is given by

$$
\Omega=\Phi(1) \Phi(1)^{\prime}=\Xi+\Theta+\Theta^{\prime}
$$


where $\Xi=\lim _{T \rightarrow \infty} \frac{1}{T} \sum_{t=1}^{T} \mathrm{E}\left(f_{t} f_{t}^{\prime}\right)$ and $\Theta=\lim _{T \rightarrow \infty} \frac{1}{T} \sum_{t=1}^{T} \mathrm{E}\left(f_{t} F_{t-1}^{\prime}\right)$. Furthermore, an invariance principle holds such that

$$
T^{-1 / 2} F_{\lfloor r T\rfloor} \Longrightarrow B_{F}(r) \text { as } T \rightarrow \infty
$$

where $B_{F}$ is a $k$-vector Brownian motion with covariance matrix $\Omega$. Assumptions $2(i)$ and $2(i i)$ are standard assumptions for factor models and ensure that the factor loadings are identifiable. Assumption 2(iii) is needed for the spurious regression results when the nonstationarity in the data is only driven by the common factors. Assumption 3(iii) specifies that a panel functional central limit theorem holds for $S_{i, t}=\sum_{s=1}^{t} e_{i, t}$, which corresponds to $E_{i, t}$ in case the idiosyncratic components are non-stationary as in (4), or its cumulative sum if (3) is true. The long-run covariance of $S_{i, t}$ is given by

$$
\Psi_{i}=\Gamma_{i}(1) \Sigma_{i} \Gamma_{i}(1)^{\prime}=\Upsilon_{i}+\Delta_{i}+\Delta_{i}^{\prime}
$$

where $\Upsilon_{i}=\lim _{T \rightarrow \infty} \frac{1}{T} \sum_{t=1}^{T} \mathrm{E}\left(e_{i, t} e_{i, t}^{\prime}\right)$ and $\Delta_{i}=\lim _{T \rightarrow \infty} \frac{1}{T} \sum_{t=1}^{T} \mathrm{E}\left(e_{i, t} S_{i, t-1}^{\prime}\right)$, and an invariance principle ensures that

$$
T^{-1 / 2} S_{i,\lfloor r T\rfloor} \Longrightarrow B_{i}(r) \text { as } T \rightarrow \infty,
$$

where $B_{i}$ is a randomly scaled $(m+1)$-vector Brownian motion with covariance matrix $\Psi_{i}$. Assumption 3(iv) ensures that the idiosyncratic terms do not cointegrate in case these are $\mathrm{I}(1)$ vectors.

The implications of these assumptions are best understood by considering the BeveridgeNelson (BN) decomposition for $F_{t}$ and for $E_{i, t}=\sum_{s=1}^{t} e_{i, s}$ :

$$
\begin{aligned}
F_{t} & =\Phi(1) \sum_{s=1}^{t} \eta_{s}+\Phi^{*}(L)\left(\eta_{t}-\eta_{0}\right)+F_{0}, \\
E_{i, t} & =\Gamma_{i}(1) \sum_{s=1}^{t} \varepsilon_{i, s}+\Gamma_{i}^{*}(L)\left(\varepsilon_{i, t}-\varepsilon_{i, 0}\right)+E_{i, 0},
\end{aligned}
$$

where $\Phi^{*}(L)=\sum_{j=0}^{\infty} \Phi_{j}^{*} L^{j}$ with $\Phi_{j}^{*}=-\sum_{l=j+1}^{\infty} \Phi_{l}, \Gamma_{i}^{*}(L)=\sum_{j=0}^{\infty} \Gamma_{i, j}^{*} L^{j}$ with $\Gamma_{i, j}^{*}=$ $-\sum_{l=j+1}^{\infty} \Gamma_{i, l}, \Phi^{*}(L)\left(\eta_{t}-\eta_{0}\right)$ and $\Gamma_{i}^{*}(L)\left(\varepsilon_{i, t}-\varepsilon_{i, 0}\right)$ are stationary with finite fourth order moments and $F_{0}$ and $E_{i, 0}$ are $O_{p}(1)$ by assumption.

If (3) is true the idiosyncratic data components are $\mathrm{I}(0)$, and the I(1) trends of the common factors contained in $\Lambda_{i} \Phi(1) \sum_{s=1}^{t} \eta_{s}$ drive the non-stationarity in the data. Then, we might observe cross-member cointegration between some $Y_{i, t}$ and $Y_{j, t}$, and between some $X_{i, t}$ and $X_{j, t}$ for some $i, j, i \neq j$, the exact cointegration structure depending on the individual loadings. The assumption on the block-diagonal structure of the factor loadings $\Lambda_{i}$ in turn implies that we have separation in a cointegrating system, see Hecq, Palm and Urbain (2002). Note that cointegration between $Y_{i, t}$ and $X_{i, t}$ would only be possible if the common factors $F_{t}^{Y}$ and $F_{t}^{X}$ would cointegrate, which is ruled out by Assumption 1 from which the full rank of the long-run covariance matrix of $F_{t}$ follows.

When $E_{i, t}$ is given by (4), both common and idiosyncratic data components are nonstationary and drive the non-stationarity in $Z_{i, t}, i=1, \ldots, N$. Furthermore, the idiosyncratic components do not cointegrate along the cross-section. Hence, we do not have cointegration "within" units, e.g. between $Y_{i, t}$ or $X_{i, t}$. The BN decomposition of the $Z_{i, t}$ is easily ob- 
tained from (1) and (7-8) and shows that the non-stationarity of $Z_{i, t}$ stems from the term $\Lambda_{i} \Phi(1) \sum_{s=1}^{t} \eta_{s}+\Gamma_{i}(1) \sum_{s=1}^{t} \varepsilon_{i, s}$.

Remark 1. The purpose of this paper is to investigate tests for no-cointegration so that we need to maintain the assumption there does exist a full column rank matrix $\beta_{i}$ such that $\beta_{i}^{\prime} Z_{i t} \sim \mathrm{I}(0)$. The model enables us however to distinguish a variety of different cases. We will concentrate on two different important cases, namely one with cross-member cointegration where we have I(1) common factors and I(0) idiosyncratic terms and one where the panel units contain common stochastic trends, but do not cointegrate even along the cross-sectional dimension so that both the common and the idiosyncratic components are I(1).

Remark 2. Strictly speaking, with I(1) common factors as well as I(1) idiosyncratic components, nothing would preclude to have different sets of cointegrating vectors that would annihilate the idiosyncratic and the common I(1) stochastic trends, see also the discussion in Grégoire (2005), Breitung and Pesaran (2005). Combining (1) and (5)-(6), the resulting $\mathrm{BN}$ representation of $Z_{i, t}$ shows that it will not be easy to annihilate both. In particular, cointegrating vector(s), say $\delta^{\prime}$, that annihilate the common $\mathrm{I}(1)$ components should lie in the left null space of $\Lambda_{i}$ such that $\delta^{\prime} \Lambda_{i} \Phi(1)=0$ while those for the idiosyncratic components, say $\beta^{\prime}$ would have to lie in the left null space of $\Gamma_{i}(1)$ such that $\beta_{i}^{\prime} \Gamma_{i}(1)=0$. If the intersection of these left null spaces is the empty set, then there does not exist a cointegrating relationship that would annihilate both the unit roots from the common stochastic trends and those of the idiosyncratic terms. This would also represent a situation where none of the $Z_{i, t}$ vectors are cointegrated. The components taken in isolation could be cointegrated though. This case is not considered in the methodological part of the paper as it would present a different probabilistic structure. Note however that the empirical analysis procedure proposed in Section 3 remains valid in this case too.

Remark 3. A similar framework is also, independently of the present work, proposed by Dees, di Mauro, Pesaran and Smith (2005) for the study of macroeconomic linkages within the Euro area. The purpose of their work is however different as no attempt to discuss tests for cointegration is made. This work is complementary to theirs.

\section{The behavior of panel residual-based tests}

The purpose of this section is to study, given the set-up introduced in the preceding section, the asymptotic behavior of some standard and popular panel tests for no-cointegration. We can view this section as providing some complementary results to the simulation results reported by Banerjee et al. (2004). The statistics we consider are designed to test for the presence of a single cointegration relationship between $Y_{i, t}$ and $X_{i, t} \cdot{ }^{1}$ Kao (1999) considers a homogenous cointegrating vector, whereas Pedroni (1999) allows for some heterogeneity. However, both rely on the cross-sectional independence of the panel unit to derive asymptotic normality for their test statistics.

\footnotetext{
${ }^{1}$ This is a restrictive assumption that we however will make in the sequel by assuming the existence of a single cointegrating vector. Approaches that allow for more than one cointegrating vectors, are reviewed in Breitung and Pesaran (2005).
} 


\subsection{Kao (1999)}

Kao (1999) proposes to estimate the homogeneous cointegrating relationship by pooled regression allowing for individual fixed effects. The regression equation is given by

$$
Y_{i, t}=\alpha_{i}+\beta X_{i, t}+u_{i, t}
$$

and the least squared dummy variable (LSDV) estimator for $\beta$ is

$$
\tilde{\beta}=\left(\sum_{i=1}^{N} \sum_{t=1}^{T} \tilde{Y}_{i, t} \tilde{X}_{i, t}^{\prime}\right)\left(\sum_{i=1}^{N} \sum_{t=1}^{T} \tilde{X}_{i, t} \tilde{X}_{i, t}^{\prime}\right)^{-1},
$$

where $\tilde{Y}_{i, t}=Y_{i, t}-\frac{1}{T} \sum_{s=1}^{T} Y_{i, s}$ and $\tilde{X}_{i, t}=X_{i, t}-\frac{1}{T} \sum_{s=1}^{T} X_{i, s}$. The residuals from this first stage regression $\tilde{u}_{i, t}=\tilde{Y}_{i, t}-\tilde{\beta} \tilde{X}_{i, t}$ will still contain a unit root under the null hypothesis of no cointegration. We now estimate a pooled DF regression

$$
\Delta \tilde{u}_{i, t}=(\rho-1) \tilde{u}_{i, t-1}+v_{i, t}
$$

where the pooled ordinary least squares (POLS) estimator of $(\rho-1)$ is given by

$$
(\tilde{\rho}-1)=\left(\sum_{i=1}^{N} \sum_{t=1}^{T} \Delta \tilde{u}_{i, t} \tilde{u}_{i, t-1}\right)\left(\sum_{i=1}^{N} \sum_{t=1}^{T} \tilde{u}_{i, t-1}^{2}\right)^{-1} .
$$

Kao (1999) tests are based on $\tilde{\rho}$ and the corresponding $t$-statistic

$$
t_{\tilde{\rho}}=(\tilde{\rho}-1)\left(\hat{s}_{\tilde{u}}^{2}\left(\sum_{i=1}^{N} \sum_{t=1}^{T} \tilde{u}_{i, t-1}^{2}\right)^{-1}\right)^{-\frac{1}{2}},
$$

where $\hat{s}_{\tilde{u}}^{2}=N^{-1} T^{-1} \sum_{i=1}^{N} \sum_{t=1}^{T} \Delta \tilde{u}_{i, t-1} \Delta \tilde{u}_{i, t-1}^{\prime}$, corrected for endogeneity and serial correlation. When the panel units are cross-sectionally independent, the test statistics are asymptotically normally distributed as $T \rightarrow \infty$ followed by $N \rightarrow \infty$. However, for the model given by (1), (2) and (3) or (4), this assumption is clearly violated. Using the results reported in Lemmas 1-3 in Appendix A, we obtain the following limit results, where $\operatorname{vec}\left(\int \mathbf{d} B_{F \Lambda} B_{F \Lambda}^{\prime}\right)=$ $\check{\Lambda} \operatorname{vec}\left(\int \mathbf{d} B_{F} B_{F}^{\prime}\right), \operatorname{vec}\left(\Theta_{F \Lambda}\right)=\check{\Lambda} \operatorname{vec}(\Theta), \operatorname{vec}\left(\int B_{F \Lambda} B_{F \Lambda}^{\prime}\right)=\check{\Lambda} \operatorname{vec}\left(\int B_{F} B_{F}^{\prime}\right), \operatorname{vec}\left(\int \mathbf{d} B_{F \Lambda} \tilde{B}_{F \Lambda}^{\prime}\right)=$ $\check{\Lambda} \operatorname{vec}\left(\int \mathbf{d} B_{F} \tilde{B}_{F}^{\prime}\right)$ and $\operatorname{vec}\left(\int \tilde{B}_{F \Lambda} \tilde{B}_{F \Lambda}^{\prime}\right)=\check{\Lambda} \operatorname{vec}\left(\int \tilde{B}_{F} \tilde{B}_{F}^{\prime}\right)$, and $\check{\Lambda}=\operatorname{plim}_{N \rightarrow \infty} \frac{1}{N} \sum_{i=1}^{N}\left(\Lambda_{i} \otimes \Lambda_{i}\right)$, and $B_{F}$ and $B_{i}$ are given in Equations (5) and (6), respectively.

Proposition 1 Given Assumptions 1, 2, 3 and 4:

(A) Consider the model given by (1) (2) and (3),

(a) $\tilde{\beta} \Longrightarrow\left(\int \tilde{B}_{F \Lambda}^{Y} \tilde{B}_{F \Lambda}^{X^{\prime}}\right)\left(\int \tilde{B}_{F \Lambda}^{X} \tilde{B}_{F \Lambda}^{X^{\prime}}\right)^{-1}=\tilde{\mathbf{b}}_{A}$ as $N, T \rightarrow \infty$ sequentially,

(b) $T(\tilde{\rho}-1) \Longrightarrow \frac{\left(1,-\tilde{\mathbf{b}}_{A}\right)\left(\int \mathbf{d} B_{F \Lambda} \tilde{B}_{F \Lambda}^{\prime}+\Theta_{F \Lambda}+\gamma_{1}-\Upsilon\right)\left(1,-\tilde{\mathbf{b}}_{A}\right)^{\prime}}{\left(1,-\tilde{\mathbf{b}}_{A}\right)\left(\int \tilde{B}_{F \Lambda} \tilde{B}_{F \Lambda}^{\prime}\right)\left(1,-\tilde{\mathbf{b}}_{A}\right)^{\prime}}$ as $T, N \rightarrow \infty$ sequentially, where $\gamma_{1}=\mathrm{E}\left(\gamma_{i 1}\right)$ and $\gamma_{i 1}=\mathrm{E}\left(\tilde{e}_{i, t-1} \tilde{e}_{i, t}^{\prime}\right)$,

(c) $t_{\tilde{\rho}}$ diverges at rate $\sqrt{N}$ as $T, N \rightarrow \infty$ sequentially.

(B) Consider the model given by (1) (2) and (4),

(a) $\tilde{\beta} \Longrightarrow\left(\int \tilde{B}_{F \Lambda}^{Y} \tilde{B}_{F \Lambda}^{X^{\prime}}+\frac{1}{6} \Psi^{Y X}\right)\left(\int \tilde{B}_{F \Lambda}^{X} \tilde{B}_{F \Lambda}^{X^{\prime}}+\frac{1}{6} \Psi^{X X}\right)^{-1}=\tilde{\mathbf{b}}_{B}$ as $N, T \rightarrow \infty$ sequentially, 
(b) $T(\tilde{\rho}-1) \Longrightarrow \frac{\left(1,-\tilde{\mathbf{b}}_{B}\right)\left(\int \mathbf{d} B_{F \Lambda} \tilde{B}_{F \Lambda}^{\prime}+\Theta_{F \Lambda}-\frac{1}{2} \Psi+\Delta\right)\left(1,-\tilde{\mathbf{b}}_{B}\right)^{\prime}}{\left(1,-\tilde{\mathbf{b}}_{B}\right)\left(\int \tilde{B}_{F \Lambda} \tilde{B}_{F \Lambda}^{\prime}+\frac{1}{6} \Psi\right)\left(1,-\tilde{\mathbf{b}}_{B}\right)^{\prime}}$ as $T, N \rightarrow \infty$ sequentially,

(c) $t_{\tilde{\rho}}$ diverges at rate $\sqrt{N}$ as $T, N \rightarrow \infty$ sequentially.

Proof: see Appendix B.

The results summarized in Proposition 1 are clearly in contrast to the asymptotic normality which Kao (1999) derives for the tests statistics for independent panels, although we have not yet considered corrections for serial correlation and endogenous regressors. Results $(A)(a)$ and $(B)(a)$ are similar to those derived by Urbain (2004) for the pooled least squares estimator (PLS). This is in sharp contrast with the $\sqrt{N}$ consistency of the LSDV estimator in the case of a spurious regression estimated from independent panel data, see Phillips and Moon (1999). The statistics proposed by Kao (1999) rely on this consistency, namely on the fact that $\tilde{\beta} \stackrel{p}{\longrightarrow} \Psi^{Y X} \Psi^{X X-1}$ where $\Psi^{Y X}$ is the average long-run covariance between $X_{i, t}$ and $Y_{i, t}$ and $\Psi^{X X}$ is the average long covariance matrix of the $X_{i, t}$ 's. The presence of common factors destroys this property and consequently also destroys the asymptotic normality of these estimators and of the statistics relying on this result. For the case of stationary idiosyncratic components, our findings are similar to the spurious regression results from time-series analysis. With non-stationary idiosyncratic components we obtain some mixture of time-series and panel spurious regression results in the limiting distributions. It is apparent that the tests are inconsistent when the data has a common factor structure, and size distortions have to be expected which will increase with $N$. The nuisance parameters in the limiting distributions given in Proposition 1 introduced by the serial correlation in the common factors and idiosyncratic components can be corrected non-parametrically for, i.e. the composite effect of $\Theta_{F \Lambda}+\gamma_{1}-\Upsilon$ or $\Theta_{F \Lambda}+\Delta$ can be accounted for. However, it is not possible to identify nuisance parameters associated with the common factors or the idiosyncratic components individually. So, the covariance of $\tilde{B}_{F \Lambda}$ as well as the average long-run covariance matrix of idiosyncratic stochastic trends, $\Psi$, will in general remain in the limits.

\subsection{Pedroni (1999)}

Pedroni (1999) allows for some heterogeneity in the cointegration relationship. He proposes to estimate a first stage regression individually for each panel member to obtain an estimate of $\beta_{i}$ from the following cointegrating equation

$$
Y_{i, t}=\alpha_{i}+\beta_{i} X_{i, t}+u_{i, t}
$$

We now have for each panel unit

$$
\tilde{\beta}_{i}=\left(\sum_{t=1}^{T} \tilde{Y}_{i, t} \tilde{X}_{i, t}^{\prime}\right)\left(\sum_{t=1}^{T} \tilde{X}_{i, t} \tilde{X}_{i, t}^{\prime}\right) .
$$

Pedroni (1999) proposes two classes of statistics, namely those based on the within-dimension denoted as "panel" statistics, and those based on the between-dimension denoted as "group mean" statistics. For the former group, the residuals from the first stage regression, $\tilde{u}_{i, t}=$ $\tilde{Y}_{i, t}-\tilde{\beta}_{i} \tilde{X}_{i, t}$, are stacked and a pooled DF regression is estimated as in (10). ${ }^{2}$ The group mean

\footnotetext{
${ }^{2}$ Note that although the estimated DF equation is the same for Kao (1999) and Pedroni (1999), the residuals used in estimation are obtained differently.
} 
statistics are based on averages of individual unit root statistics, derived from

$$
\Delta \tilde{u}_{i, t}=\left(\rho_{i}-1\right) \tilde{u}_{i, t-1}+v_{i, t}
$$

to obtain

$$
\left(\tilde{\rho}_{i}-1\right)=\left(\sum_{t=1}^{T} \Delta \tilde{u}_{i, t} \tilde{u}_{i, t-1}\right)\left(\sum_{t=1}^{T} \tilde{u}_{i, t-1}^{2}\right)^{-1} .
$$

Consider now the panel-rho statistic denoted by $Z_{\tilde{\rho}_{N T}-1}$ and the group-mean rho statistic $\tilde{Z}_{\tilde{\rho}_{N T}-1}$ given by

$$
Z_{\tilde{\rho}_{N T}-1}=\left(\sum_{i=1}^{N} \sum_{t=2}^{T}\left(\Delta \tilde{u}_{i, t} \tilde{u}_{i, t-1}-\hat{\boldsymbol{\lambda}}_{i}\right)\right)\left(\sum_{i=1}^{N} \sum_{t=1}^{T} \tilde{u}_{i, t-1}^{2}\right)^{-1}
$$

and

$$
\tilde{Z}_{\tilde{\rho}_{N T}-1}=\sum_{i=1}^{N}\left(\left(\sum_{t=2}^{T}\left(\Delta \tilde{u}_{i, t} \tilde{u}_{i, t-1}-\hat{\boldsymbol{\lambda}}_{i}\right)\right)\left(\sum_{t=1}^{T} \tilde{u}_{i, t-1}^{2}\right)^{-1}\right),
$$

with $\hat{\boldsymbol{\lambda}}_{i}=T^{-1} \sum_{s=1}^{J} \omega_{s J} \sum_{t=s+1}^{T} \tilde{v}_{i, t} \tilde{v}_{i, t-s}$ where $\tilde{v}_{i, t}$ are the residuals of the second stage regression, and $J$ and $\omega_{s J}$ are suitable bandwidth and kernel function, respectively. For these 2 statistics, we obtain the following limiting results:

Proposition 2 Given Assumptions 1, 2, 3 and 4:

(A) Consider the model given by (1) to (3),

(a) $\tilde{\beta}_{i} \Longrightarrow\left(\lambda_{1 i}^{\prime}\left(\int \tilde{B}_{F}^{Y} \tilde{B}_{F}^{X^{\prime}} \lambda_{2 i}\right)\left(\lambda_{2 i}^{\prime}\left(\int \tilde{B}_{F}^{X} \tilde{B}_{F}^{X^{\prime}}\right) \lambda_{2 i}\right)^{-1}=\tilde{\mathbf{b}}_{i A}\right.$ as $T \rightarrow \infty$

(b) $T Z_{\tilde{\rho}_{N T}-1} \Longrightarrow \frac{\sum_{i=1}^{N} \lambda_{1 i}^{\prime} L_{11}^{\prime} \int \mathbf{d} Q_{F} \tilde{Q}_{F}^{\prime} L_{11} \lambda_{1 i}}{\sum_{i=1}^{N} \lambda_{1 i}^{\prime} L_{11}^{\prime} \int \tilde{Q}_{F} \tilde{Q}_{F}^{\prime} L_{11} \lambda_{i 1}}$ as $T \rightarrow \infty$,

(c) $T \tilde{Z}_{\tilde{\rho}_{N T}-1} \Longrightarrow \sum_{i=1}^{N} \frac{\lambda_{1 i}^{\prime} L_{11}^{\prime} \int \mathbf{d} Q_{F} \tilde{Q}_{F}^{\prime} L_{11} \lambda_{1 i}}{\lambda_{1 i}^{\prime} L_{11}^{\prime} \int \tilde{Q}_{F} \tilde{Q}_{F}^{\prime} L_{11} \lambda_{i 1}}$ as $T \rightarrow \infty$,

where $\tilde{Q}=\tilde{W}_{F}^{Y}-\left(\int \tilde{W}_{F}^{Y} \tilde{W}_{F}^{X \prime}\right)\left(\int \tilde{W}_{F}^{X} \tilde{W}_{F}^{X \prime}\right)^{-1} \tilde{W}_{F}^{X}, \tilde{W}_{F}$ is a demeaned $k$-vector standard Brownian motion, and $L_{11}$ is upper left element of $L$, the block-triangular decomposition of $\Omega=L^{\prime} L$.

(B) Consider the model given by (1) to (4),
(a)
$\tilde{\beta}_{i} \Longrightarrow$
$\left(\lambda_{1 i}^{\prime} \int \tilde{B}_{F}^{Y} \tilde{B}_{F}^{X^{\prime}} \lambda_{2 i}+\int \tilde{B}_{i}^{Y} \tilde{B}_{i}^{X^{\prime}}+\lambda_{1 i}^{\prime} \int \tilde{B}_{F}^{Y} \tilde{B}_{i}^{X^{\prime}}+\int \tilde{B}_{i}^{Y} \tilde{B}_{F}^{X^{\prime}} \lambda_{2 i}\right)$
$\left(\lambda_{2 i}^{\prime} \int \tilde{B}_{F}^{X} \tilde{B}_{F}^{X^{\prime}} \lambda_{2 i}+\int \tilde{B}_{i}^{X} \tilde{B}_{i}^{X^{\prime}}+\lambda_{2 i}^{\prime} \int \tilde{B}_{F}^{X} \tilde{B}_{i}^{X^{\prime}}+\int \tilde{B}_{i}^{X} \tilde{B}_{F}^{X^{\prime}} \lambda_{2 i}\right)^{-1}=\tilde{\mathbf{b}}_{i B}$
as $T \rightarrow \infty$,
(b) $T Z_{\tilde{\rho}_{N T}-1} \Longrightarrow \frac{\sum_{i=1}^{N}\left(1-\tilde{\mathbf{b}}_{i B}\right)\left(\Lambda_{i}^{\prime}\left(\int \mathbf{d} B_{F} \tilde{B}_{F}\right) \Lambda_{i}^{\prime}+\int \mathbf{d} B_{i} \tilde{B}_{i}^{\prime}+\Lambda_{i} \int \mathbf{d} B_{F} \tilde{B}_{i}+\int \mathbf{d} B_{i} \tilde{B}_{F}^{\prime} \Lambda_{i}^{\prime}\right)\left(1-\tilde{\mathbf{b}}_{i B}\right)^{\prime}}{\sum_{i=1}^{N}\left(1-\tilde{\mathbf{b}}_{i B}\right)\left(\Lambda_{i} \int \tilde{B}_{F} \tilde{B}_{F}^{\prime} \Lambda_{i}^{\prime}+\int \tilde{B}_{i} \tilde{B}_{i}^{\prime}+\Lambda_{i} \int \tilde{B}_{F} \tilde{B}_{i}^{\prime}+\int \tilde{B}_{i} \tilde{B}_{F}^{\prime} \Lambda_{i}^{\prime}\right)\left(1-\tilde{\mathbf{b}}_{i B}\right)^{\prime}}$ as $T \rightarrow \infty$, (c) $T \tilde{Z}_{\tilde{\rho}_{N T}-1} \Longrightarrow \sum_{i=1}^{N} \frac{\left(1-\tilde{\mathbf{b}}_{i B}\right)\left(\Lambda_{i}^{\prime}\left(\int \mathbf{d} B_{F} \tilde{B}_{F}\right) \Lambda_{i}^{\prime}+\int \mathbf{d} B_{i} \tilde{B}_{i}^{\prime}+\Lambda_{i} \int \mathbf{d} B_{F} \tilde{B}_{i}+\int \mathbf{d} B_{i} \tilde{B}_{F}^{\prime} \Lambda_{i}^{\prime}\right)\left(1-\tilde{\mathbf{b}}_{i B}\right)^{\prime}}{\left(1-\tilde{\mathbf{b}}_{i B}\right)\left(\Lambda_{i} \int \tilde{B}_{F} \tilde{B}_{F}^{\prime} \Lambda_{i}^{\prime}+\int \tilde{B}_{i} \tilde{B}_{i}^{\prime}+\Lambda_{i} \int \tilde{B}_{F} \tilde{B}_{i}^{\prime} \iint \tilde{B}_{i} \tilde{B}_{F}^{\prime} \Lambda_{i}^{\prime}\right)\left(1-\tilde{\mathbf{b}}_{i B}\right)^{\prime}}$ as
$\quad T \rightarrow \infty$

Proof: see Appendix C.

For the panel-rho and group-mean-rho statistics Pedroni $(1999,2004)$ derives asymptotic normality when they are properly standardized. In particular, $\sqrt{N} T Z_{\tilde{\rho}_{N T}-1}-\sqrt{N} \theta_{2} \theta_{1}^{-1}$ and 
$N^{-\frac{1}{2}} T \tilde{Z}_{\tilde{\rho}_{N T}-1}-\sqrt{N} \tilde{\theta}_{1}$ are asymptotically normally distributed for independent panels, where $\theta_{1}, \theta_{2}$ and $\tilde{\theta}_{1}$ are means of functionals of Brownian motions (for details see Pedroni, 2004). The results from Proposition 2 indicate that under the DGP we consider, $T Z_{\tilde{\rho}_{N T}-1}$ and $N^{-1} T \tilde{Z}_{\tilde{\rho}_{N T}-1}$ converge, so that the two test-statistics diverge at rate $\sqrt{N}$ when standardized as above. Furthermore, due to the presence of the common factors, the individual statistics will not be independent along the cross-section, so that the use of a CLT to derive asymptotic normality of the average statistic will be invalid. The result is similar to that derived by Lyhagen (2000) for the Im-Pesaran-Shin (IPS) statistics. Also, for independent panels the distributions of $Z_{\tilde{\rho}_{N T}-1}$ and $\tilde{Z}_{\tilde{\rho}_{N T}-1}$ will be nuisance parameter free. For the DGP we consider, this is not true in general. Although the composite effect of serial correlation in the the common factors and idiosyncratic components can be corrected for non-parametrically, nuisance parameters coming only from the common factors or from the idiosyncratic components cannot be identified. So, the limiting distributions will in general depend on the long-run covariances of the common and/or idiosyncratic stochastic trends. A special case arises when there is a single common factor in $Y_{i, t}$ and the idiosyncratic components are stationary. Then, $\lambda_{1 i} L_{11}$ will cancel from the limits given in Proposition $2 \mathrm{~A}(\mathrm{~b})$ and (c).

\section{A two-step procedure to test for (no)cointegration in the presence of common factors}

The previous section shows that standard panel tests for the null of cointegration suffer from serious theoretical problems when applied to data that have been generated by a common factor structure. In this section, we address the problem using a simple approach based on the Bai and Ng (2004) PANIC methodology. ${ }^{3}$

Note that a related, albeit different, idea is exploited in the recent work of Banerjee and Carrion-i-Silvestre (2005). These authors assume a factor structure for the disturbance of a panel static regression model:

$$
\begin{aligned}
Y_{i, t} & =\alpha_{i}+\beta_{i} X_{i, t}+u_{i, t} \\
u_{i, t} & =\gamma_{i}^{\prime} F_{t}+E_{i, t},
\end{aligned}
$$

where $F_{t}$ and $E_{i, t}$ are the common factors and the idiosyncratic components respectively that can be either I(1) or I(0). A similar framework is used by Bai and Kao (2004) for the estimation of a cointegrating relationship in the presence of common factors. Under strong exogeneity of $X_{i, t}$ for all $i$, this framework leads to panel statistics for the null of no-cointegration that have the same distribution as those for panel unit root tests and hence are not affected by the number of regressors. ${ }^{4}$ This framework makes it however difficult to interpret the case of no-cointegration (spurious regression) if the regressors $X_{i, t}$ also have a factor structure since in the absence of cointegration, assuming a factor structure for the residuals essentially boils down to assuming a factor structure for $Y_{i, t}$ only. The restrictiveness of the exogeneity assumption should also be underlined.

The route we follow in this paper is slightly different and based on the framework presented in Section 2. We assume that the data are generated by the approximate factor structure

\footnotetext{
${ }^{3}$ Wagner and Müller-Fürstenberger (2004) use similar ideas in an empirical study of the Kuznets curve.

${ }^{4} \mathrm{~A}$ similar set-up is retained by Westerlund (2005) who proposes Durbin-Hausman tests for cointegration in panels.
} 
(1)-(4). Since the behavior of the statistics has been shown in the previous section to depend essentially on the location of the unit roots in the factors and/or in the idiosyncratic components, a testing strategy needs to be chosen that covers the two important cases of interest.

The basic idea of the procedure we propose is to exploit results derived in Bai and $\mathrm{Ng}$ (2004) for their PANIC methodology. In particular, under the DGP (1)-(4), and using the partitioning of $Z_{i, t}=\left(Y_{i, t}, X_{i, t}^{\prime}\right)^{\prime}$, both $Y_{i, t}$ and $X_{i, t}$ are characterized by a factor structure. Since the problems encountered with the usual panel cointegration tests stem from the unit root in the factors, we propose a simple procedure that essentially consists in two different steps.

Step 1. Conduct a preliminary PANIC analysis of each variable $X_{i, t}$ and $Y_{i, t}$ individually to extract the common factors, using for example the principal components approach advocated by Bai and $\mathrm{Ng}$ (2004). Test for unit roots in both the factors and the idiosyncratic components using the approach proposed by Bai and $\mathrm{Ng}$ (2004) or using a related panel unit root tests valid in the presence of a factor structure such as the one proposed by Breitung and Das (2005).

Step 2. a. If I(1) common factors and I(0) idiosyncratic components are detected, then we face the situation of cross-member cointegration and consequently the non-stationarity in the panel is entirely due to a reduced number of common stochastic trends. Cointegration between $Y_{i, t}$ and $X_{i, t}$ can only occur if the common factors for $Y_{i, t}$ cointegrate with those of $X_{i, t}$. The null of no-cointegration between these estimated factors can be tested using a Johansen type of likelihood ratio test for example.

b. If I(1) common factors and I(1) idiosyncratic components are detected, similarly to Banerjee and Carrion-i-Silvestre (2005), we will work with defactored series. In contrast to their work however, instead of defactoring the residuals from a static regression we will defactor separately $Y_{i, t}$ and $X_{i, t}$. The defactored $Y_{i, t}$ (e.g. the estimated idiosyncratic components) is simply obtained as $\widehat{E}_{i, t}^{Y}=\sum_{s=1}^{t} \widehat{e}_{i, s}^{Y}=$ $\sum_{s=1}^{t}\left(\Delta Y_{i, s}-\widehat{\lambda}_{1, i}^{\prime} \widehat{f}_{s}\right)$ where $\widehat{f}_{s}$ is a consistent factor estimate of $f_{t}$ in $(2)$ and $\widehat{\lambda}_{1, i}^{\prime}$ a consistent estimate of the loading. ${ }^{5}$ The same defactoring procedure is applied to the $X_{i, t}$ 's.

Testing for no-cointegration between the defactored data (the estimated idiosyncratic components) can be conducted using standard panel tests for no-cointegration such as those of Pedroni $(1999,2004)$ while testing for the absence of cointegration between the common factors (the I(1) common trends) can again be performed using Johansen's likelihood ratio type tests.

The rejection of no-cointegration between $Y_{i, t}$ and $X_{i, t}$ only occurs if both statistics reject.

One should make a few remarks at this stage.

Remark 4. The theoretical justification and motivation for this sequential procedure is analogous to the one behind the PANIC approach for panel unit root analysis. In particular, since the DGP implies that all series have a Bai and Ng (2004) representation, we will

\footnotetext{
${ }^{5}$ In the case of a single factor, the moment estimator defactoring approach of Phillips and Sul (2003) which does not require large $N$ could also be used.
} 
proceed by analogy with the results derived in Bai and $\mathrm{Ng}(2004)^{6}$, which exploits the fact that, provided the number of common factors is known or consistently selected using one of the asymptotically consistent model selection procedures discussed in Bai and $\mathrm{Ng}$ (2004), then it holds that $T^{-1 / 2} \sum_{s=2}^{t} \hat{e}_{i, s}^{Y}=T^{-1 / 2} \sum_{s=2}^{t} e_{i, s}^{Y}+O_{p}\left(C_{N T}^{-1}\right)$ where $\hat{e}_{i, t}^{Y}$ is the estimated idiosyncratic component (e.g. the defactored variables), $\hat{e}_{i, t}^{Y}=\Delta Y_{i, s}-\widehat{\lambda}_{1, i}^{\prime} \widehat{f}_{s}, \widehat{f}_{s}$ a consistent factor estimate of $f_{t}, \widehat{\lambda}_{1, i}^{\prime}$ a consistent estimate of the loading and $C_{N T}^{-1}=\min \left(N^{1 / 2}, T^{1 / 2}\right)$. It holds consequently that $\frac{1}{\sqrt{T}} \sum_{t=1}^{\lfloor T r\rfloor} \hat{e}_{i, t}^{Y} \Longrightarrow B_{i}^{Y}(r), \forall i$ where $B_{i}^{Y}(r)$ is the first elements of the $(m+1)$-vector Brownian motion $B_{i}(r) . B_{i}^{Y}(r)$ and $B_{j}^{Y}(r)$ are furthermore uncorrelated Brownian motions for $i \neq j$. The same holds for $X_{i, t}$.

Consequently, standard panel no-cointegration tests derived under the maintained assumption of independent panel unit, such as those proposed by Pedroni (2004) for example, can be used on the defactored observations.

Remark 5. This approach requires both large $N$ and $T$ which is one of the important limitation. Notice, also that this approach will have finite sample properties that can, at best, be close to those observed for the tests when applied to a panel data set with independent units. This will be analyzed in the next subsection by Monte Carlo simulations.

Remark 6. If the rank of the long-run covariance matrix of the factors turns out to be smaller than $k$, that is if the factors cointegrate, then a further step is needed to assess overall lack of cointegration between $Y_{i, t}$ and $X_{i, t}$. No cointegration then requires separability in cointegration as discussed and analyzed in details in Hecq et al. (2002).

\section{Some Monte Carlo Evidence}

The approach we propose in the preceding section has several characteristics that call for a Monte Carlo analysis of some of its finite sample properties. In particular, the theoretical foundation requires both large $N$ and $T$ which is not always met in typical applications of panel cointegration techniques. We will focus on the empirical size properties of the proposed approach, namely testing for no-cointegration using "defactored" data, as it was shown that tests designed for cross-sectionally independent data may suffer from dramatic size distortions when applied to panel with cross-member cointegration for example as pointed out by Banerjee et al. (2004). The DGP is a simple bivariate process that obeys to the representation (1)-(4) and is given by

$$
\begin{aligned}
Z_{i, t} & =\Lambda_{i} F_{t}+E_{i, t}, \quad E_{i, t}=\left\{\begin{array}{l}
e_{i, t} \\
E_{i, t-1}+e_{i, t}
\end{array},\right. \\
e_{i, t} & =\varepsilon_{i, t}+\Gamma_{i} \varepsilon_{i, t-1} \\
F_{t} & =F_{t-1}+f_{t}, \\
f_{t} & =\eta_{t}+\Phi_{1} \eta_{t-1},
\end{aligned}
$$

\footnotetext{
${ }^{6}$ A similar result is given in Kapetanios (2004, Theorem 2).
} 
where $\varepsilon_{i, t} \sim$ i.i.d. $N\left(0, \Sigma_{i}\right), \eta_{t} \sim$ i.i.d. $N\left(0, I_{2}\right)$. The loading matrix has a diagonal structure

$$
\Lambda_{i}=\left[\begin{array}{cc}
\lambda_{1 i} & 0 \\
0 & \lambda_{2 i}
\end{array}\right]
$$

with $\lambda_{1 i}, \lambda_{2 i} \sim U[-1,3]$ where $U$ denotes uniform distributions. The remaining parameters are also drawn from independent uniform distributions to allow for some degree of heterogeneity: $\Phi_{11,22} \sim U[0.5,0.7], \Phi_{12,21} \sim U[0,0.5], \Sigma_{i, 11,22} \sim U[1,1.4], \Gamma_{11,22} \sim U[0.5,0.7], \Phi_{12,21} \sim$ $U[0,0.5]$ and $\Sigma_{i, 12,21} \sim U[0,0.2]$. The sample size has been set to $T=\{25,50,100\}$ and the number of units in the panel is set to $N=\{25,50,100\}$. We consider the rejection frequencies based on 2000 replications $^{7}$ for the following statistics:

1. Kao's pooled normalized coefficient (the $\rho$ test) test based on the raw data,

2. Kao's pooled ADF test based on the raw data,

3. Pedroni's panel- $t$ statistics based on raw data,

4. Pedroni's panel- $\rho$ statistics based on raw data,

5. Pedroni's group mean $t$ statistics based on raw data,

6. Pedroni's group mean $\rho$ statistics based on raw data,

7. Pedroni's group mean $t$ statistics based on defactored data,

8. Pedroni's group mean $\rho$ statistics based on defactored data,

9. Johansen trace test for the estimated common factors, using the information criterion of Aznar and Salvador (2002) to select the lag length of the VECM.

For the last two statistics based on the defactored data, we assume the number of factors $k$ known in the simulations. For the ADF type statistics the lag length was selected using the AIC. For the non-parametric correction for serial correlation, we used a quadratic spectral kernel with a bandwidth of $3.21 T^{\frac{1}{3}}$ as proposed in Andrews (1991).

The two polar cases that we consider in the simulations are the cases discussed earlier namely:

- the case of cross-member cointegration in which the common factors are I(1) and the idiosyncratic component are $\mathbf{I}(0)$,

- the case where both common factors and idiosyncratic components are I(1).

Table 1 and Table 2 report the rejection frequencies for both cases but where we have in addition excluded any serial correlation in $e_{t}$ and in $f_{t}$. All statistics considered in these two tables are then also constructed without correction for serial correlation. Tables 3-4 report rejection frequencies for the general case with short-run dynamics modelled as MA(1) processes as mentioned above.

As expected from the results of the previous section, the usual panel statistics perform bady in cases of cross-member cointegration where the nonstationary is essentially due to the

\footnotetext{
${ }^{7}$ All experiments are carried out using GAUSS 6.0.
} 
common factors. In these cases, as was already observed in Banerjee et al. (2004), standard tests assuming uncorrelated units are very severely oversized even for cases where $N$ and $T$ are both equal to 100. A common features of all four tables that is worth underlining is the superiority of the group mean statistics compared to their (pooled) panel versions. When both the common factors and the idiosyncratic components are I(1) but we exclude serial correlation from the DGP (Table 2), we observe that the distributions for Kao's $D F_{\rho}$ and $D F_{t}$ statistics diverge to the right. Therefore, these statistics have rejection frequencies of 0 in that case.

\section{INSERT TABLES 1 TO 4 ABOUt HERE}

The last three rows of the tables are the rejection frequencies of the panel tests using the defactored data (column 2-7) and of the system test for the estimated factors (columns 810). These are denoted by Idiosyncratic Panel-t, Idiosyncratic Group- $\rho$ and Aznar/Johansen. Table 1 and 3 report the frequencies for the case of cross-member cointegration where the generated idiosyncratic components are $\mathbf{I}(0)$. As could be expected, the tests on the defactored data always reject the null since these components are stationary. The tests of no-cointegration between the extracted common factors have correct size in the case without short-run dynamics and are slightly oversized when MA(1) dynamics is introduced since the rejection frequencies vary from 0.14 to 0.08 for a nominal size of 0.05 . The panel cointegration tests applied to the defactored data, i.e. the estimated idiosyncratic components, is undersized in the case where both components are I(1) and where short-run dynamics is present (see Table 4).

Tables 5 to 7 present some simulation results for cases with cointegration in some data component, but without serial correlation in the error terms. In particular, the cases we consider are cointegration in $F_{t}$ but not in $E_{i, t}$ (Table 5), cointegration in $E_{i, t}$ for all $i$ but not in $F_{t}$ (Table 6), and cointegration in $F_{t}$ and $E_{i, t}$ for all $i$ with a common cointegrating vector (Table 7 ). Note that only in the later case, $Y_{i, t}$ and $X_{i, t}$ cointegrate for all $i$. Both Kao test statistics we consider do not reject the null of no cointegration when only the common factors cointegrate. When cointegration is only present in the idiosyncratic component, Kao's normalized coefficient test is over-sized, while the ADF test has rejection frequencies close to the nominal $5 \%$ level. When cointegration is present in either the common or the idiosyncratic component, all Pedroni tests are strongly over-sized, with slightly lower size distortions if cointegration is only present in $E_{i, t}$. In that case, also the group mean statistics are less distorted than the panel ones. Test statistics for the defactored data are slightly under-sized if there is no cointegration in the idiosyncratic component, while they correctly reject the null if $E_{i, t}^{Y}$ and $E_{i, t}^{X}$ are cointegrated. The trace test for the estimated common factors has a size close to the nominal $5 \%$ when the common factors do not cointegrate, and a high rejection frequency of the full rank null hypothesis for the long-run covariance matrix of $F_{t}$ if there is cointegration present in the common factors. If $Y_{i, t}$ and $X_{i, t}$ cointegrate, all tests have a power of (close to) 1.

\section{InSERT TABLES 5 TO 7 ABOUt HERE}

Tables 8 to 10 consider similar cases as Tables 5 to 7 , but with serial correlation introduced in the error terms. When either $F_{t}$ or $E_{i, t}$ is cointegrated, and hence there is no cointegration in $Z_{i, t}$, the test statistics by Kao and Pedroni are oversized with size distortions increasing in $N$ and $T$. The panel cointegration tests applied to the defactored data are undersized when 
$E_{i, t}$ is not cointegrated, but have a power of 1 for almost all combinations of $N$ and $T$ when there is cointegration in the idiosyncratic component. The trace test applied to the extracted common factors is slightly oversized when no cointegration is present in the common factors, and has high power when $F_{t}^{Y}$ and $F_{t}^{X}$ cointegrate.

INSERT TABLES 8 TO 10 ABOUT HERE

\section{Empirical Illustration}

Among the economic hypotheses that are selected to illustrate the feasibility of new techniques in non-stationary econometrics, the purchasing power parity (PPP) hypothesis is without any doubts among the most popular ones. This applications was also chosen in Pedroni (2004) to illustrate the feasibility of his tests. In his illustration, he also highlights the importance of taking cross-sectional dependence into account even if this was outside the scope of his initial study. Since PPP is a field where persistent cross-sectional dependence is present in the data due to the very nature of the economic problems studied (see Lyhagen, 2000; Banerjee et al, 2004), we will also consider PPP to illustrate the approach discussed earlier in this paper. Following Pedroni (2004), we will focus on a version of the hypothesis known as weak longrun PPP which posits that although nominal exchange rates and aggregate price ratios may move together over long periods, there are reasons to think that in practice the movements may not be perfectly proportional. Motivations for this deviation from the perfect PPP hypothesis are numerous and will not be discussed here (transportation costs, measurement errors, productivity differential, ....). All these factors however speak in favor of allowing for substantial heterogeneity, since under the alternative hypothesis of cointegration there are no reasons to expect the cointegrating vector to be the same for all countries.

The empirical model adopted can be written as

$$
s_{i, t}=\alpha_{i}+\beta_{i} p_{i, t}+\epsilon_{i, t},
$$

where $s_{i, t}$ is the log nominal bilateral US dollar exchange rate at time $t$ for country $i$ and $p_{i, t}$ is the log price level differential between country $i$ and the United States at time $t$. A rejection of the null hypothesis of no cointegration in this equation is taken as empirical evidence in favor of the weak PPP hypothesis.

We employ quarterly (in contrast to Pedroni who uses both monthly and annual IFS data) on nominal exchange rates and consumer price index (CPI) deflators for the postBretton Woods/pre-Euro period from the 1st quarter of 1974 to 3rd quarter of 1998 . The crosssection consists of 18 countries: Australia, Austria, Belgium, Canada, Denmark, Finland, France, Germany, Ireland, Italy, Japan, The Netherlands, Norway, Portugal, Spain, Sweden, Switzerland and the UK. For the Kao-ADF statistics the lag length was selected using the AIC. For the non-parametric correction used in the construction of the Pedroni statistics and the Kao- $\rho^{*}$ statistics, we used a quadratic spectral kernel with a bandwidth of $3.21 T^{\frac{1}{3}}$.

\section{INSERT TABLES 11-12 ABOUT HERE}

The results are reported in Table 8 . Although not reported to save space, ${ }^{8}$, we note that the number of rejections of the null of no-cointegration when using the individual time series

\footnotetext{
${ }^{8}$ The results are available upon request from the authors.
} 
statistics is too low to favor weak PPP. A similar observation is made in Pedroni (2004). When we consider the standard panel tests applied to the raw data, we see that the panel tests of Kao clearly reject the null. The same holds for the "within" panel tests of Pedroni, while the group-mean statistics do not reject, globally only lending some weak support to the PPP hypothesis. The aforementioned tests do however not take into account any cross-sectional dependence.

Following the procedure proposed in Section 3 we first consider a Bai and $\mathrm{Ng}$ (2004) type PANIC analysis for the series for $s_{i, t}$ and $p_{i, t}$ separately. The number of common factors is selected using Bai and Ng's (2002) IC1 criterion. For both the exchange rates and the prices a single common factor is selected. The pooled p-value Fisher type unit root tests on the extracted idiosyncratic components do not reject the null hypothesis (the statistics take the value -2.25 and -1.46 respectively). Similarly, the ADF tests for the extracted common factors do not reject the unit root hypothesis since these take the value -2.79 and -2.63 respectively for $\hat{F}_{Y}$ and $\hat{F}_{X}$ which is larger than the $5 \%$ critical of -3.416 . Given these outcomes, the second step of the procedure consists in testing for no-cointegration between the idiosyncratic components as well as testing for the absence of cointegration between the estimated factors. The results are reported in Table 9. As it is clear from the entries in the Table, none of the reported statistics reject the null of no-cointegration.

\section{Conclusions}

In this paper we have considered the problem of testing for (no-)cointegration in panel data set characterized by strong permanent cross-sectional dependencies that take the form of an approximate factor representation inspired by the work of Bai and $\mathrm{Ng}$ (2004). We focus on two polar cases that we believe are of empirical relevance namely the case of cross-member cointegration and the case where the panel units have both common and individual specific stochastic trends that are not cointegrated.

For both classes of DGP's, we study analytically the behavior of several popular tests for panel cointegration including Kao (1999) and Pedroni's (1999, 2004) residual-based panel nocointegration tests that have been widely used in empirical work over the recent years. The results complement and help to understand some of the Monte Carlo reported by Banerjee et al. (2004b). For example, when the number of common factors generating the non-stationarity in the panel is kept fixed while the dimension of the panel increases, then the Gaussian limiting results derived for the independent case are not valid anymore. Tests that are based on pooled or LSDV estimation of the underlying panel cointegration static regression may in some cases diverge with $\sqrt{N}$ and hence important size distortions are to be expected already in panels with moderate cross-sectional dimension. Group mean statistics are also affected and not asymptotically gaussian anymore.

Given these observations, we propose a simple two-step procedure to address the issue of cointegration testing in panels with common factors. Specifically, we propose to first conduct a PANIC analysis of each series, to defactor the data if $\mathrm{I}(1)$ common factors are found and then to conduct "standard" panel cointegration analysis on defactored data. This approach is similar in spirit to the recent work of Banerjee and Carrion-i-Silvestre (2005) but in contrast to their work strong exogeneity assumptions are not required. One of its advantages is that it covers many sub-cases of interest and allows to have a clear picture of the common/global and iniosyncratic/individual specific components in the panel. The procedure is simple to 
apply and makes use of existing tools. Some simulation results show that the procedure we propose seems to have reasonable size properties.

While being attractive due, among other things, to its ease of application and nice properties, some limitations inherent in this approach should be mentioned. A first limitation of the proposed procedure, as well as of that proposed by Banerjee and Carrion-i-Silvestre (2005), is that the theoretical validity relies on both large $N$ and large $T$ which may be unrealistic for applications with "moderate" $N$ and large $T$. The performance of the proposed procedures, in particular the power properties, in such situation still needs to be further studied even if the size properties reported in Monte Carlo section are promising. If considering large $N$ analysis is clearly inappropriate for the problem under study, then an alternative would be to follow the work of Demetrescu and Tarcolea (2005) who propose a non-linear IV testing approach or to consider the use of bootstrapping techniques that seem to work well from an empirical point of view (see Fachin, 2005). Future work should study the relative merits of these different approaches both theoretically and empirically.

A second limitation worth mentioning lies in the fact that the approach is a residual-based testing procedure and hence suffers from the usual critiques against residual-based tests such as the maintained assumptions of a single cointegrating relationship (if it exists) as well as the imposition of the common factor restriction. Nothing however precludes to conceptually extend the idea developed in this paper to other cointegration techniques that would not suffer from these drawbacks.

\section{References}

[1] Andrews, D.W.K. (1991). Heteroskedasticity and Autocorrelation Consistent Covariance Matrix Estimation, Econometrica 59, No.3, 817-858.

[2] Andrews, D.W.K. and Monahan, C. (1992). An Improved Heteroskedasticity and Autocorrelation Consistent Covariance Matrix Estimator, Econometrica 60, No.4, 953-966.

[3] Aznar, A. and Salvador, M. (2002). Selecting the Rank of the Cointegration Space and the Form of the Intercept Using an Information Criterion, Econometric Theory 18, 926947.

[4] Bai, J. and Kao, C. (2004). On the Estimation and Inference of a Panel Cointegration Model with Cross-Sectional Dependence, mimeo, Presented at ESEM2004.

[5] Bai, J. and Ng, S. (2004). A PANIC Attack on Unit Roots and Cointegration, Econometrica 72 , No.4, 1127-1177.

[6] Banerjee, A. and Carrion-i-Silvestre, J.L. (2005). Breaking Panel Data Cointegration, mimeo.

[7] Banerjee, A., Marcellino, M. and Osbat, C. (2004). Some Cautions on the Use of Panel Methods for Integrated Series of Macro-Economic Data, Econometrics Journal 7, 2, 322-340.

[8] Banerjee, A., Marcellino, M. and Osbat, C. (2005). Testing for PPP: Should We Use Panel Methods?, Empirical Economics 30(1), 77-91. 
[9] Breitung, J. and Das, S. (2005). Testing for Unit Roots in Panels With a Factor Structure, University of Bonn, unpublished.

[10] Breitung, J. and Pesaran, H.M. (2005). Unit Roots and Cointegration in Panels, forthcoming in Matyas, L. and Sevestre, P. (eds.) The Econometrics of Panel Data, Kluwer Academic Publishers, (forthcoming).

[11] Davidson, J. and de Jong, R. (2000). The Functional Central Limit Theorem and Weak Convergence to Stochastic Integrals I: Weakly Dependent Processes, Econometric Theory $16,621-642$.

[12] Dees, S., F. di Mauro, M. H. Pesaran and Smith, L.V. (2005). Exploring the International Linkages of the Euro Area: A Global VAR Analysis, CESifoWorking Paper No. 1425. Presented at CESifo Area Conference on Macro, Money and International Finance, February 2005.

[13] Demetrescu, M. and Tarcolea, A.-I. (2005). Panel Cointegration Testing using Nonlinear Instruments, Working Paper, Goethe-University Frankfurt.

[14] Fachin, S. (2005), Long-Run Trends in Internal Migrations in Italy: a Study in Panel Cointegration with Dependent Units, mimeo, University of Rome 'La Sapienza'.

[15] Gegenbach, C., Palm, F.C. and Urbain, J.-P. (2004). Panel Unit Root Tests in the Presence of Cross-Sectional Dependencies: Comparison and Implications for Modelling, METEOR Research Memorandum 04039 Universiteit Maastricht.

[16] Grégoire, S. (2005). Representation and Statistical Analysis of Weakly Linearly Exchangeable Dynamic Panels, Working Paper, CREST.

[17] Hecq, A., Palm, F.C. and Urbain, J.-P. (2002). Separation, Weak Exogeneity and PT Decomposition in Cointegrated VAR Systems with Common Features, Econometric Reviews 21, 273-307.

[18] Im, K.S., Pesaran, M.H. and Shin, Y. (2003). Testing for Unit Roots in Heterogenous Panels, Journal of Econometrics 115, 53-74.

[19] Kao, C. (1999). Spurious Regression and Residual-Based Tests for Cointegration in Panel Data, Journal of Econometrics 90, 144

[20] Kapetanios, G. (2004). Dynamic Factor Extraction of Cross-Sectional Dependence in Panel Unit Root Tests, Working Paper 509, Queen Mary College, University of London.

[21] Levin, A., Lin, C.F. and Chu C.S.J. (2002). Unit Root Tests in Panel Data: Asymptotic and Finite-Sample Properties, Journal of Econometrics 108, 1-24.

[22] Lyhagen, J. (2000), Why not Use Standard Panel Unit Root Test for Testing PPP, mimeo, Stockholm School of Economics.

[23] Moon, H.R. and Perron, B. (2004). Testing for a Unit Root in Panels with Dynamic Factors, Journal of Econometrics 122, 81-126.

[24] Pedroni, P. (1999). Critical Values for Cointegration Tests in Heterogeneous Panels with Multiple Regressors, Oxford Bulletin of Economics and Statistics, 61, 653-670. 
[25] Pedroni, P. (2004). Panel Cointegration, Asymptotic and Finite Sample Properties of Pooled Time Series tests with an Application to the PPP hypothesis, Econometric Theory 20, 597-625.

[26] Pedroni, P. and Urbain, J.-P. (2001). Cross Member Cointegration in Non-Stationary Panels, mimeo, Universtiteit Maastricht.

[27] Pesaran, M.H. (2003). A Simple Panel Unit Root Test in the Presence of Cross Section Dependence, mimeo, Cambridge University.

[28] Phillips, P.C.B. and Durlauf, S. (1986). Multiple Time Series Regression with Integrated Processes, Review of Economic Studies 53, 473-495.

[29] Phillips, P.C.B. and Moon, H.R. (1999). Linear Regression Limit Theory for Nonstationary Panel Data, Econometrica 67, 1057-1111.

[30] Phillips, P. and Sul, D. (2003). Dynamic Panel Estimation and Homogeneity Testing Under Cross Section Dependence, Econometrics Journal 6, 217-259.

[31] Urbain, J.-P. (2004). Spurious Regression in Nonstationary Panels with Cross-Member Cointegration, mimeo, Presented at ESEM2004.

[32] Wagner,M. and Müller-Fürstenberger, G. (2004). The Carbon Kuznets Curve: A Cloudy Picture Emitted by Bad Econometrics?, Discussion paper 4.18, University of Bern.

[33] Westerlund, J. (2005). New Simple Tests for Panel Cointegration, Working Paper 2005:8, Lund University, Department of Economics. 


\section{A Proofs of Lemmas 1 to 3}

Given Assumptions 1 to 4, we can summarize some convergence results. In the following lemmas, for a variable $x_{t}, \tilde{x}_{t}=x_{t}-\frac{1}{T} \sum_{t=1}^{T} x_{t}$ is the demeaned value. Similarly, for a Brownian motion $B$, $\tilde{B}=B-\int B$ denotes the demeaned Brownian motion. Furthermore, for non-random factor loadings, $\bar{\Lambda}=\lim _{N \rightarrow \infty} \frac{1}{N} \sum_{i=1}^{N} \Lambda_{i}$, while for random factor loadings $\bar{\Lambda}=\mathrm{E}\left(\Lambda_{i}\right), \Psi=\mathrm{E}\left(\Psi_{i}\right)$ and $\Delta=\mathrm{E}\left(\Delta_{i}\right)$.

\section{A.1 Lemma 1: Common Component}

Lemma 1 Given Assumptions 1, 2 and 4,

$$
\frac{1}{T} \sum_{t=1}^{T} \Lambda_{i} f_{t} F_{t-1}^{\prime} \Lambda_{i}^{\prime} \quad \Longrightarrow \quad \Lambda_{i}\left(\int \mathbf{d} B_{F} B_{F}^{\prime}+\Theta\right) \Lambda_{i}^{\prime} \quad \text { as } T \rightarrow \infty \text {, and }
$$
$\frac{1}{N} \sum_{i=1}^{N} \Lambda_{i}\left(\int \mathbf{d} B_{F} B_{F}^{\prime}+\Theta\right) \Lambda_{i}^{\prime} \stackrel{p}{\longrightarrow} \int \mathbf{d} B_{F \Lambda} B_{F \Lambda}^{\prime}+\Theta_{F \Lambda} \quad$ as $N \rightarrow \infty$,

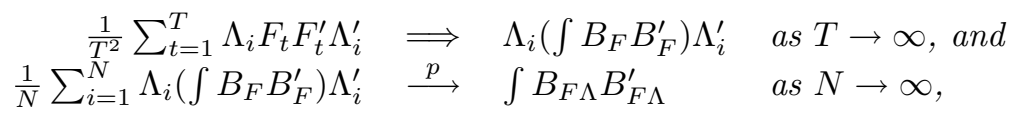

(c) $\quad \frac{1}{T} \sum_{t=1}^{T} \Lambda_{i} f_{t} \tilde{F}_{t-1}^{\prime} \Lambda_{i}^{\prime} \quad \Longrightarrow \quad \Lambda_{i}\left(\int \mathbf{d} B_{F} \tilde{B}_{F}^{\prime}+\Theta\right) \Lambda_{i}^{\prime} \quad$ as $T \rightarrow \infty$, and $\frac{1}{N} \sum_{i=1}^{N} \Lambda_{i}\left(\int \mathbf{d} B_{F} \tilde{B}_{F}^{\prime}+\Theta\right) \Lambda_{i}^{\prime} \quad \stackrel{p}{\longrightarrow} \int \mathbf{d} B_{F \Lambda} \tilde{B}_{F \Lambda}^{\prime}+\Theta_{F \Lambda} \quad$ as $N \rightarrow \infty$,

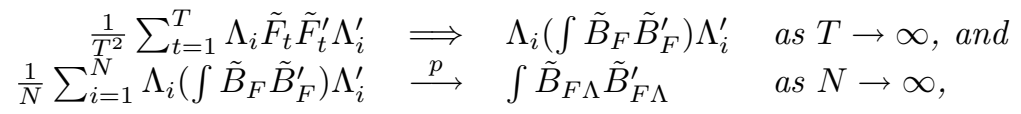

where $\operatorname{vec}\left(\int \mathbf{d} B_{F \Lambda} B_{F \Lambda}^{\prime}\right)=\check{\Lambda} \operatorname{vec}\left(\int \mathbf{d} B_{F} B_{F}^{\prime}\right), \operatorname{vec}\left(\Theta_{F \Lambda}\right)=\check{\Lambda} \operatorname{vec}(\Theta), \operatorname{vec}\left(\int B_{F \Lambda} B_{F \Lambda}^{\prime}\right)=\check{\Lambda} \operatorname{vec}\left(\int B_{F} B_{F}^{\prime}\right)$, $\operatorname{vec}\left(\int \mathbf{d} B_{F \Lambda} \tilde{B}_{F \Lambda}^{\prime}\right)=\check{\Lambda} \operatorname{vec}\left(\int \mathbf{d} B_{F} \tilde{B}_{F}^{\prime}\right)$ and $\operatorname{vec}\left(\int \tilde{B}_{F \Lambda} \tilde{B}_{F \Lambda}^{\prime}\right)=\check{\Lambda} \operatorname{vec}\left(\int \tilde{B}_{F} \tilde{B}_{F}^{\prime}\right)$, and $\check{\Lambda}=\operatorname{plim}_{N \rightarrow \infty} \frac{1}{N} \sum_{i=1}^{N}\left(\Lambda_{i} \otimes \Lambda_{i}\right)$.

\section{A.2 Proof of Lemma 1}

For the common factors given in (2) we find the following Beveridge-Nelson (BN) decomposition:

$$
F_{t}=\Phi(1) \sum_{s=1}^{t} \eta_{s}+\Phi^{*}(L)\left(\eta_{t}-\eta_{0}\right)+F_{0}
$$

where $\Phi^{*}(L)=\sum_{j=0}^{\infty} \Phi_{j}^{*} L^{j}$ with $\Phi_{j}^{*}=-\sum_{l=j+1}^{\infty} \Phi_{l}$. Now, $\frac{1}{\sqrt{T}} \Phi(1) \sum_{s=1}^{\lfloor r T\rfloor} \eta_{s} \Longrightarrow \Phi(1) W_{F}(r) \equiv B_{F}(r)$ by the FCLT, where $W_{F}$ is standard Brownian Motion. Furthermore, $\Phi^{*}(L)\left(\eta_{t}-\eta_{0}\right)$ is stationary with finite fourth order moments such that $\frac{1}{\sqrt{T}} \Phi^{*}(L)\left(\eta_{t}-\eta_{0}\right) \stackrel{p}{\longrightarrow} 0$, and $F_{0}$ is $O_{p}(1)$ by assumption.

(a) We have $\frac{1}{T} \sum_{t=1}^{T} \Lambda_{i} f_{t} F_{t-1}^{\prime} \Lambda_{i}^{\prime}=\Lambda_{i}\left(\frac{1}{T} \sum_{t=1}^{T} f_{t} F_{t-1}^{\prime}\right) \Lambda_{i}^{\prime}$. Now, $\frac{1}{T} \sum_{t=1}^{T} f_{t} F_{t-1}^{\prime} \Longrightarrow \int \mathbf{d} B_{F} B_{F}^{\prime}+$ $\Theta$ as $T \rightarrow \infty$ as shown in e.g. Davidson and de Jong (2000), and the result of Lemma 1 (a) follows immediately. Furthermore, $\operatorname{vec}\left(\Lambda_{i}\left(\int \mathbf{d} B_{F} B_{F}^{\prime}+\Theta\right) \Lambda_{i}^{\prime}\right)=\left(\Lambda_{i} \otimes \Lambda_{i}\right) \operatorname{vec}\left(\int \mathbf{d} B_{F} B_{F}^{\prime}+\right.$ $\Theta)$. As $\mathrm{E}\left\|\left(\Lambda_{i} \otimes \Lambda_{i}\right)\right\|^{2}=\mathrm{E}\left\|\Lambda_{i}\right\|^{4} \leq M$ by Assumption 2 (i), we can apply a LLN to $\frac{1}{N} \sum_{i=1}^{N}\left(\Lambda_{i} \otimes \Lambda_{i}\right)$. Denote $\operatorname{plim}\left(\Lambda_{i} \otimes \Lambda_{i}\right)=\check{\Lambda}$ to obtain the second result of Lemma 1 (a).

(b) The proof of Lemma 1 (b) is similar to that of (a), except that $\frac{1}{T^{2}} \sum F_{t} F_{t}^{\prime} \Longrightarrow \int B_{F} B_{F}^{\prime}$ as shown in e.g. Phillips and Durlauf (1986) in the first step.

(c) $\frac{1}{T} \sum_{t=1}^{T} f_{t} \tilde{F}_{t-1}^{\prime}=\frac{1}{T} \sum_{t=1}^{T} f_{t} F_{t-1}^{\prime}-\left(\sum_{t=1}^{T} \frac{f_{t}}{\sqrt{T}}\right)\left(\frac{1}{T^{\frac{3}{2}}} \sum_{s=1}^{T} F_{s}^{\prime}\right)$. Now, $\frac{1}{T} \sum_{t=1}^{T} f_{t} F_{t-1}^{\prime} \Longrightarrow$ $\int \mathbf{d} B_{F} B_{F}^{\prime}+\Theta$ while $\left(\sum_{t=1}^{T} \frac{f_{t}}{\sqrt{T}}\right)\left(\frac{1}{T^{\frac{3}{2}}} \sum_{s=1}^{T} F_{s}^{\prime}\right) \Longrightarrow \int \mathbf{d} B_{F}\left(\int B_{F}\right)^{\prime}$ as $T \rightarrow \infty$, so that $\frac{1}{T} \sum_{t=1}^{T} f_{t} \tilde{F}_{t-1}^{\prime} \Longrightarrow \int \mathbf{d} B_{F} \tilde{B}_{F}^{\prime}+\Theta$. The remainder of the proof follows the same arguments as above.

(d) Now, $\sum_{t=1}^{T} \tilde{F}_{t} \tilde{F}_{t}^{\prime} \Longrightarrow \int \tilde{B}_{F} \tilde{B}_{F}^{\prime}$ as shown in Phillips and Moon (1999), and the limit as $N \rightarrow \infty$ follows as above. 


\section{A.3 Lemma 2: Idiosyncratic Components}

\section{Lemma 2 Given Assumption 3,}

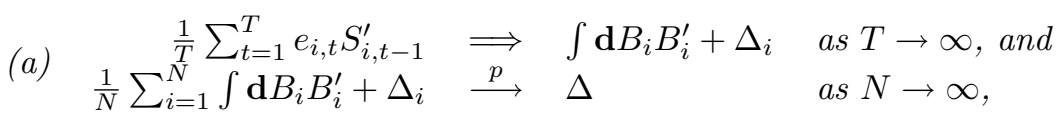

$\begin{array}{llll}\frac{1}{T^{2}} \sum_{t=1}^{T} S_{i, t} S_{i, t}^{\prime} & \Longrightarrow & \int B_{i} B_{i}^{\prime} & \text { as } T \rightarrow \infty \text {, and } \\ \frac{1}{N} \sum_{i=1}^{N} \int B_{i} B_{i}^{\prime} & \longrightarrow & \frac{1}{2} \Psi & \text { as } N \rightarrow \infty,\end{array}$

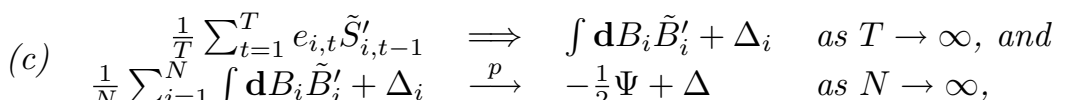

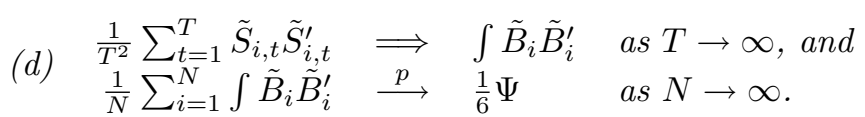

\section{A.4 Proof of Lemma 2}

For the partial sum process $S_{i, t}=\sum_{s=1}^{t} e_{i, s}$ we obtain a BN decomposition

$$
S_{i, t}=\Gamma_{i}(1) \sum_{s=1}^{t} \varepsilon_{i, s}+\Gamma_{i}^{*}(L)\left(\varepsilon_{i, t}-\varepsilon_{i, 0}\right)+E_{i, 0},
$$

where $\Gamma_{i}^{*}(L)=\sum_{j=0}^{\infty} \Gamma_{i, j}^{*} L^{j}$ with $\Gamma_{i, j}^{*}=-\sum_{l=j+1}^{\infty} \Gamma_{i, l}$. Now, $\frac{1}{\sqrt{T}} S_{i,\lfloor r T\rfloor} \Longrightarrow \Gamma_{i}(1) \sum_{i}^{\frac{1}{2}} W_{i}(r) \equiv B_{i}(r)$ as $T \rightarrow \infty$ for all $i$, where $W_{i}$ is standard Brownian motion and $\Sigma_{i}^{\frac{1}{2}}$ is the Cholesky decomposition of $\Sigma_{i}$ such that $\Sigma_{i}^{\frac{1}{2}} \Sigma_{i}^{\frac{1}{2} \prime}=\Sigma_{i}$ as shown in Phillips and Moon (1999). Furthermore, $B_{i}$ and $B_{j}$ are i.i.d over the $i$-dimension.

(a) We have $\frac{1}{T} \sum_{t=1}^{T} e_{i, t} S_{i, t-1}^{\prime} \Longrightarrow \int \mathbf{d} B_{i} B_{i}^{\prime}+\Delta_{i}$ as $T \rightarrow \infty$ as shown in Davidson and de Jong (2000). Now, $\int \mathbf{d} B_{i} B_{i}^{\prime}$ are i.i.d across the $i$-dimension with $\mathrm{E}\left(\int \mathbf{d} B_{i} B_{i}^{\prime}\right)=0$ and $E\left\|\operatorname{vec}\left(\int \mathbf{d} B_{i} B_{i}^{\prime}\right)\right\|^{2}<M$. So, we can apply a LLN to find $\frac{1}{N} \sum_{i=1}^{N} \int \mathbf{d} B_{i} B_{i}^{\prime} \stackrel{p}{\longrightarrow} 0$. Furthermore, a LLN also applies such that $\sum_{i=1}^{N} \Delta_{i} \stackrel{p}{\longrightarrow} \Delta \equiv \mathrm{E}\left(\Delta_{i}\right)$, which proves the first result.

(b) This result is proven in Phillips and Moon (1999).

(c) $\frac{1}{T} \sum_{t=1}^{T} e_{i, t} \tilde{S}_{i, t-1}^{\prime}=\frac{1}{T} \sum_{t=1}^{T} e_{i, t} S_{i, t-1}^{\prime}-\frac{1}{T} \sum_{t=1}^{T} e_{i, t} \bar{S}_{i}^{\prime}$, where $\bar{S}_{i}^{\prime}=\frac{1}{T} \sum_{t=1}^{T} S_{i, t}$. Now, $\frac{1}{T} \sum t=1^{T} e_{i, t} S_{i, t-1}^{\prime} \Longrightarrow \int \mathbf{d} B_{i} B_{i}^{\prime}+\Delta_{i}$, while $\frac{1}{T} \sum_{t=1}^{T} e_{i, t} \bar{S}_{i}^{\prime}=\frac{1}{\sqrt{T}} S_{T} \frac{1}{T^{\frac{3}{2}}} \sum_{t=1}^{T} S_{i, t}^{\prime} \Longrightarrow$ $B_{i}(1) \int B_{i}$ as $T \rightarrow \infty$. So, $\frac{1}{T} \sum_{t=1}^{T} e_{i, t} \tilde{S}_{i, t-1}^{\prime} \Longrightarrow \int \mathbf{d} B_{i} \tilde{B}_{i}^{\prime}+\Delta_{i}$ as $T \rightarrow \infty$. Furthermore, $\mathrm{E}\left(\int \mathbf{d} B_{i} \tilde{B}_{i}^{\prime}\right)=-\frac{1}{2} \Psi$ and hence, using similar arguments as in (a) $\frac{1}{N} \sum_{i=1}^{N} \int \mathbf{d} B_{i} \tilde{B}_{i}^{\prime}+\Delta_{i} \stackrel{p}{\longrightarrow}$ $-\frac{1}{2} \Psi+\Delta$ as $N \rightarrow \infty$.

(d) See Phillips and Moon (1999).

\section{A.5 Lemma 3}

Lemma 3 Given Assumptions 1, 2, 3 and 4

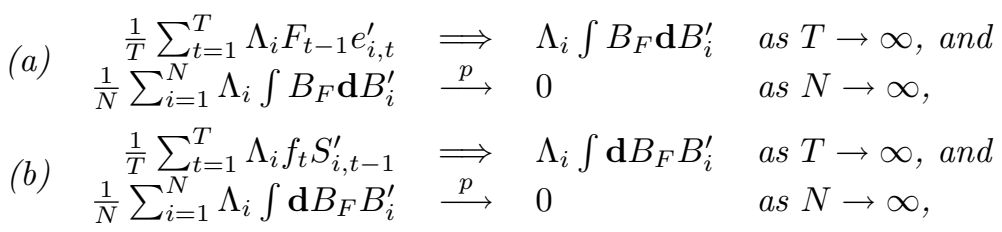

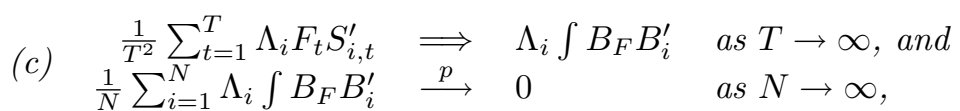

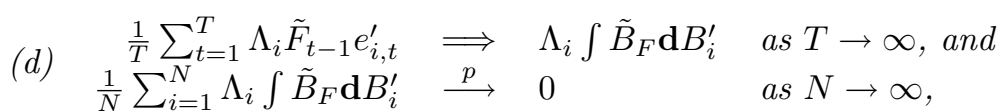



(e)
$\frac{1}{T} \sum_{t=1}^{T} \Lambda_{i} \tilde{F}_{t-1} \tilde{e}_{i, t}^{\prime} \quad \Longrightarrow \quad \Lambda_{i} \int \tilde{B}_{F} \mathbf{d} B_{i}^{\prime}$
as $T \rightarrow \infty$, and
$\frac{1}{N} \sum_{i=1}^{N} \Lambda_{i} \int \tilde{B}_{F} \mathbf{d} B_{i}^{\prime} \stackrel{p}{\longrightarrow} 0$
as $N \rightarrow \infty$,
(f)
$\frac{1}{T} \sum_{t=1}^{T} \Lambda_{i} f_{t} \tilde{S}_{i, t-1}^{\prime} \quad \Longrightarrow \quad \Lambda_{i} \int \mathbf{d} B_{F} \tilde{B}_{i}^{\prime}$
as $T \rightarrow \infty$, and
$\frac{1}{N} \sum_{i=1}^{N} \Lambda_{i} \int \mathbf{d} B_{F} \tilde{B}_{i}^{\prime} \stackrel{p}{\longrightarrow} 0$
as $N \rightarrow \infty$,
(g)
$\frac{1}{T^{2}} \sum_{t=1}^{T} \Lambda_{i} \tilde{F}_{t} \tilde{S}_{i, t}^{\prime} \quad \Longrightarrow \quad \Lambda_{i} \int \tilde{B}_{F} \tilde{B}_{i}^{\prime}$
as $T \rightarrow \infty$, and
$\frac{1}{N} \sum_{i=1}^{N} \Lambda_{i} \int \tilde{B}_{F} \tilde{B}_{i}^{\prime} \stackrel{p}{\longrightarrow} 0 \quad$ as $N \rightarrow \infty$,

\section{A.6 Proof of Lemma 3}

For each $i$, the stacked error vector $w_{i, t}=\left(f_{t}^{\prime}, e_{i, t}^{\prime}\right)^{\prime}$ and the corresponding partial sum process $W_{i, t}=\sum_{s=1}^{t} w_{i, s}=\left(F_{t}^{\prime}, S_{i, t}^{\prime}\right)^{\prime}$ fulfill the conditions for a FCLT, such that $\frac{1}{\sqrt{T}} W_{i,\lfloor r T\rfloor} \Longrightarrow B_{w_{i}}(r)=$ $\left(B_{F}(r)^{\prime}, B_{i}(r)^{\prime}\right)^{\prime}$. Due to the independence of $f_{t}$ and $e_{i, t}$, the covariance matrix of $B_{w_{i}}$ will have zero off-diagonal blocks. Now, for every panel unit $i$ we obtain time series spurious regression results as $T \rightarrow \infty$. Furthermore, the functionals of $B_{F}$ and $B_{i}$ we obtain in the first step have zero mean and finite variance, and are uncorrelated across the $i$-dimension of the panel. So, we can apply a LLN to the average to find the limits as $N \rightarrow \infty$. We present the proof for (a), (b)-(g) are obtained using a similar line of argumentation.

(a) The limit as $T \rightarrow \infty$ follows from applying a spurious regression result as above and noting that $\mathrm{E}\left(F_{t-1} e_{i, t}\right)=0$ for all $i$ and $t$. Now, taking expectations we find $\mathrm{E}\left(\Lambda_{i} \int B_{F} \mathbf{d} B_{i}\right)=0$, while $\mathrm{E}\left\|\operatorname{vec}\left(\Lambda_{i} \int B_{F} \mathbf{d} B_{i}\right)\right\|^{2}<M$. for all $i$. Applying a LLN, we find $\frac{1}{N} \sum_{i=1}^{N} \Lambda_{i} \int B_{F} \mathbf{d} B_{i} \stackrel{p}{\longrightarrow} 0$.

Lemma 1 presents convergence results for the common data component $\Lambda_{i} F_{t}$. The limiting distributions are functionals of Brownian motions weighted by the factor loadings, even as $N \rightarrow \infty$. These results are intuitive, as we assume a fixed number of common factors. Lemma 2 summarizes the convergence for the idiosyncratic components, where we recover the panel spurious regression results for Phillips and Moon (1999). In Lemma 3, the limits for the cross-products of the common and individual specific components are given. It is evident that these cross-products will only affect limiting distributions for finite $N$, but as $N \rightarrow \infty$ these effects will vanish due to the independence of the shock driving $F_{t}$ and $E_{i, t}$.

\section{B Proof of Proposition 1}

\section{B.1 Proposition 1 (a): Convergence of $\tilde{\beta}$}

The LSDV estimator of $\beta$ is given by $\tilde{\beta}=\left(\sum_{i=1}^{N} \sum_{t=1}^{T} \tilde{Y}_{i, t} \tilde{X}_{i, t}^{\prime}\right)\left(\sum_{i=1}^{N} \sum_{t=1}^{T} \tilde{X}_{i, t} \tilde{X}_{i, t}^{\prime}\right)^{-1}$. Consider the numerator

$$
\begin{aligned}
\sum_{i=1}^{N} \sum_{t=1}^{T} \tilde{Y}_{i, t} \tilde{X}_{i, t}^{\prime} & =\sum_{i=1}^{N} \sum_{t=1}^{T}\left(\lambda_{1 i}^{\prime} \tilde{F}_{t}^{Y}+\tilde{E}_{i, t}^{Y}\right)\left(\lambda_{2 i}^{\prime} \tilde{F}_{t}^{X}+\tilde{E}_{i, t}^{X}\right)^{\prime} \\
& =\sum_{i=1}^{N} \sum_{t=1}^{T}\left(\lambda_{1 i}^{\prime} \tilde{F}_{t}^{Y} \tilde{F}_{t}^{X \prime} \lambda_{21}+\tilde{E}_{i, t}^{Y} \tilde{E}_{i, t}^{X \prime}+\lambda_{1 i}^{\prime} \tilde{F}_{t}^{Y} \tilde{E}_{i, t}^{X \prime}+\tilde{E}_{i, t}^{Y} \tilde{F}_{t}^{X \prime} \lambda_{21}\right) .
\end{aligned}
$$

If the idiosyncratic term is given by (3), we have $\sum_{i=1}^{N}\left(O_{p}\left(T^{2}\right)+O_{p}(T)+O_{p}(T)+O_{p}(T)\right)$ in (18). So, as $T \rightarrow \infty, \sum_{i=1}^{N} \frac{1}{T^{2}} \sum_{t=1}^{T} \tilde{Y}_{i, t} \tilde{X}_{i, t}^{\prime} \Longrightarrow \sum_{i=1}^{N} \lambda_{1 i}^{\prime} \int \tilde{B}_{F}^{Y} \tilde{B}_{F}^{X \prime} \lambda_{2 i}$ from the first result of Lemma 1 (d). Now, using the second result we obtain $\frac{1}{N} \sum_{i=1}^{N} \lambda_{1 i}^{\prime} \int_{\tilde{B}_{F}}^{Y} \tilde{B}_{F}^{X \prime} \lambda_{2 i} \stackrel{p}{\longrightarrow} \int \tilde{B}_{F \Lambda}^{Y} \tilde{B}_{F \Lambda}^{X}{ }^{\prime}$ as $N \rightarrow \infty$, where $\int \tilde{B}_{F \Lambda}^{Y} \tilde{B}_{F \Lambda}^{X}{ }^{\prime}$ is the $1 \times m$ upper right block of $\int \tilde{B}_{F \Lambda} \tilde{B}_{F \Lambda}^{\prime}$ defined in Lemma 1 .

If the idiosyncratic terms are also I(1), such that the DGP includes (4), all terms in (18) are 
$O_{p}\left(T^{2}\right)$ when summed over $T$. Using Lemmas 1 (d), 2 (d) and 3 (g) we find as $T \rightarrow \infty$,

$$
\sum_{i=1}^{N} \frac{1}{T^{2}} \sum_{t=1}^{T} \tilde{Y}_{i, t} \tilde{X}_{i, t}^{\prime} \Longrightarrow \sum_{i=1}^{N}\left(\lambda_{1 i}^{\prime} \int \tilde{B}_{F}^{Y} \tilde{B}_{F}^{X \prime} \lambda_{2 i}+\int \tilde{B}_{i}^{Y} \tilde{B}_{i}^{X \prime}+\lambda_{1 i}^{\prime} \int \tilde{B}_{F}^{Y} \tilde{B}_{i}^{X \prime}+\int \tilde{B}_{i}^{Y} \tilde{B}_{F}^{X \prime} \lambda_{21}\right) .
$$

The terms given above are $O_{p}(N)+O_{p}(N)+o_{p}(N)+o_{p}(N)$, and we obtain

$$
\frac{1}{N} \sum_{i=1}^{N}\left(\lambda_{1 i}^{\prime} \int \tilde{B}_{F}^{Y} \tilde{B}_{F}^{X \prime} \lambda_{2 i}+\int \tilde{B}_{i}^{Y} \tilde{B}_{i}^{X \prime}+\lambda_{1 i}^{\prime} \int \tilde{B}_{F}^{Y} \tilde{B}_{i}^{X \prime}+\int \tilde{B}_{i}^{Y} \tilde{B}_{F}^{X^{\prime}} \lambda_{21}\right) \stackrel{p}{\longrightarrow} \int \tilde{B}_{F \Lambda}^{Y} \tilde{B}_{F \Lambda}^{X}{ }^{\prime}+\frac{1}{6} \Psi^{Y X}
$$

as $N \rightarrow \infty$, where $\Psi^{Y X}$ is the upper right $1 \times m$ block of $\Psi$.

Now the denominator of $\tilde{\beta}$ is given by

$$
\begin{aligned}
\sum_{i=1}^{N} \sum_{t=1}^{T} \tilde{X}_{i, t} \tilde{X}_{i, t}^{\prime} & =\sum_{i=1}^{N} \sum_{t=1}^{T}\left(\lambda_{1 i}^{\prime} \tilde{F}_{t}^{X}+\tilde{E}_{i, t}^{X}\right)\left(\lambda_{2 i}^{\prime} \tilde{F}_{t}^{X}+\tilde{E}_{i, t}^{X}\right)^{\prime} \\
& =\sum_{i=1}^{N} \sum_{t=1}^{T}\left(\lambda_{2 i}^{\prime} \tilde{F}_{t}^{X} \tilde{F}_{t}^{X \prime} \lambda_{21}+\tilde{E}_{i, t}^{X} \tilde{E}_{i, t}^{X \prime}+\lambda_{2 i}^{\prime} \tilde{F}_{t}^{X} \tilde{E}_{i, t}^{X \prime}+\tilde{E}_{i, t}^{X} \tilde{F}_{t}^{X \prime} \lambda_{21}\right) .
\end{aligned}
$$

Similar to the results for the numerator, the terms in (19) are $\sum_{i=1}^{N}\left(O_{p}\left(T^{2}\right)+O_{p}(T)+O_{p}(T)+\right.$ $O_{p}(T)$ ), if the DGP contains (3). Hence, $\sum_{i=1}^{N} \frac{1}{T^{2}} \sum_{t=1}^{T} \tilde{X}_{i, t} \tilde{X}_{i, t}^{\prime} \Longrightarrow \sum_{i=1}^{N} \lambda_{2 i}^{\prime} \int \tilde{B}_{F}^{X} \tilde{B}_{F}^{X \prime} \lambda_{2 i}$ as $T \rightarrow \infty$. Furthermore, the remaining term is $O_{p}(N)$, and we obtain $\frac{1}{N} \sum_{i=1}^{N} \frac{1}{T^{2}} \sum_{t=1}^{T} \tilde{X}_{i, t} \tilde{X}_{i, t}^{\prime} \stackrel{\mathcal{L}, p}{\longrightarrow} \int \tilde{B}_{F \Lambda}^{X} \tilde{B}_{F \Lambda}^{X}{ }^{\prime}$ as $T \rightarrow \infty$ followed by $N \rightarrow \infty$, where $\int \tilde{B}_{F \Lambda}^{X} \tilde{B}_{F \Lambda}^{X}$ 'is the lower right $m \times m$ block of $\int \tilde{B}_{F \Lambda} \tilde{B}_{F \Lambda}^{\prime}$.

If the true DGP contains (4), all terms in the summation over $T$ in (19) are $O_{p}\left(T^{2}\right)$ and we have

$$
\sum_{i=1}^{N} \frac{1}{T^{2}} \sum_{t=1}^{T} \tilde{X}_{i, t} \tilde{X}_{i, t}^{\prime} \Longrightarrow \sum_{i=1}^{N}\left(\lambda_{2 i}^{\prime} \int \tilde{B}_{F}^{X} \tilde{B}_{F}^{X \prime} \lambda_{2 i}+\int \tilde{B}_{i}^{X} \tilde{B}_{i}^{X \prime}+\lambda_{2 i}^{\prime} \int \tilde{B}_{F}^{X} \tilde{B}_{i}^{X \prime}+\int \tilde{B}_{i}^{X} \tilde{B}_{F}^{X \prime} \lambda_{21}\right),
$$

as $T \rightarrow \infty$. As above, the cross-products between common and idiosyncratic components will vanish in the cross-sectional average as $N \rightarrow \infty$, and we find

$$
\frac{1}{N} \sum_{i=1}^{N}\left(\lambda_{2 i}^{\prime} \int \tilde{B}_{F}^{X} \tilde{B}_{F}^{X \prime} \lambda_{2 i}+\int \tilde{B}_{i}^{X} \tilde{B}_{i}^{X \prime}+\lambda_{2 i}^{\prime} \int \tilde{B}_{F}^{X} \tilde{B}_{i}^{X \prime}+\int \tilde{B}_{i}^{X} \tilde{B}_{F}^{X \prime} \lambda_{21}\right) \stackrel{p}{\longrightarrow} \int \tilde{B}_{F \Lambda}^{X} \tilde{B}_{F \Lambda}^{X}{ }^{\prime}+\frac{1}{6} \Psi^{X X}
$$

as $N \rightarrow \infty$, where $\Psi^{X X}$ is the lower right $m \times m$ block of $\Psi$.

Combining the results given above yields Proposition 1 A(a) and B(a).

\section{B.2 Proposition 1 (b): Convergence of $\tilde{\rho}$}

The residuals from the first stage PLS regression are given by $\tilde{u}_{i, t}=(1-\tilde{\beta}) Z_{i, t}=Y_{i, t}-\tilde{\beta} X_{i, t}$. For the pooled regression given in (10) we have

$$
(\tilde{\rho}-1)=\left(\sum_{i=1}^{N} \sum_{t=2}^{T}(1-\tilde{\beta}) \Delta Z_{i, t} \tilde{Z}_{i, t-1}^{\prime}(1-\tilde{\beta})^{\prime}\right)\left(\sum_{i=1}^{N} \sum_{t=2}^{T}(1-\tilde{\beta}) \tilde{Z}_{i, t-1} \tilde{Z}_{i, t-1}^{\prime}(1-\tilde{\beta})^{\prime}\right)^{-1} .
$$

For the numerator consider

$$
\begin{aligned}
\sum_{i=1}^{N} \sum_{t=2}^{T} \Delta Z_{i, t} \tilde{Z}_{i, t-1}^{\prime} & =\sum_{i=1}^{N} \sum_{t=2}^{T}\left(\Lambda_{i} f_{t}+\Delta E_{i, t}\right)\left(\Lambda_{i} \tilde{F}_{t-1}+\tilde{E}_{i, t-1}\right)^{\prime} \\
& =\sum_{i=1}^{N} \sum_{t=2}^{T}\left(\Lambda_{i} f_{t} \tilde{F}_{t-1}^{\prime} \Lambda_{i}^{\prime}+\Delta E_{i, t} \tilde{E}_{i, t-1}^{\prime}+\Lambda_{i} f_{t} \tilde{E}_{i, t-1}^{\prime}+\Delta E_{i, t} \tilde{F}_{t-1}^{\prime} \Lambda_{i}^{\prime}\right) .
\end{aligned}
$$


From Lemma 1 (c), $\frac{1}{N} \sum_{i=1}^{N} \frac{1}{T} \sum_{t=2}^{T} \Lambda_{i} f_{t} \tilde{F}_{t-1} \Lambda_{i}^{\prime} \stackrel{\mathcal{L}, p}{\longrightarrow} \int \mathbf{d} B_{F \Lambda} \tilde{B}_{F \Lambda}+\Theta_{F \Lambda}$ as $T \rightarrow \infty$ followed by $N \rightarrow \infty$. If the idiosyncratic terms are I(0), i.e. the true DGP is given by (3),

$$
\sum_{i=1}^{N} \sum_{t=2}^{T} \Delta E_{i, t} \tilde{E}_{i, t-1}^{\prime}=\sum_{i=1}^{N} \sum_{t=2}^{T}\left(\left(e_{i, t}-e_{i, t-1}\right) e_{i, t-1}^{\prime}-\left(e_{i, t}-e_{i, t-1}\right) \bar{e}_{i}\right)
$$

where $\bar{e}_{i}=\frac{1}{T} \sum_{t=1}^{T} e_{i, t}$. Now, $\frac{1}{T} \sum_{t=2}^{T} e_{i, t} e_{i, t-1}^{\prime} \stackrel{p}{\longrightarrow} \gamma_{i 1}$ as $T \rightarrow \infty$, with $\gamma_{i 1}=\lim _{T \rightarrow \infty} \frac{1}{T} \sum_{i=1}^{T} \mathrm{E}\left(e_{i, t} e_{i, t-1}\right)$, and $\frac{1}{N} \sum_{i=1}^{N} \gamma_{i 1} \stackrel{p}{\longrightarrow} \gamma_{1}$ as $N \rightarrow \infty$, with $\gamma_{1} \equiv \mathrm{E}\left(\gamma_{i 1}\right)$. Also, $\frac{1}{T} \sum_{t=2}^{T} e_{i, t-1} e_{i, t-1}^{\prime} \stackrel{p}{\longrightarrow} \Upsilon_{i}$ as $T \rightarrow \infty$ and $\frac{1}{N} \sum_{i=1}^{N} \Upsilon_{i} \stackrel{p}{\longrightarrow} \Upsilon$ as $N \rightarrow \infty$. Furthermore, $\frac{1}{T} \sum_{t=2}^{T} e_{i, t} \bar{e}_{i}^{\prime} \stackrel{p}{\longrightarrow} 0$ and $\frac{1}{T} \sum_{t=2}^{T} e_{i, t-1} \bar{e}_{i}^{\prime} \stackrel{p}{\longrightarrow} 0$ as $T \rightarrow \infty$. Hence, $\frac{1}{N} \sum_{i=1}^{N} \frac{1}{T} \sum_{t=2}^{T} \Delta E_{i, t} \tilde{E}_{i, t-1}^{\prime} \stackrel{p}{\longrightarrow} \gamma_{1}-\Upsilon$ as $T \rightarrow \infty$ followed by $N \rightarrow \infty$.

For the third term in (21) we have,

$$
\begin{aligned}
\frac{1}{T} \sum_{t=2}^{T} \Lambda_{i} f_{t} \tilde{E}_{i, t-1}^{\prime} & =\frac{1}{T} \sum_{t=2}^{T} \Lambda_{i} f_{t}\left(e_{i, t-1}-\bar{e}_{i}\right)^{\prime} \\
& =\frac{1}{T} \sum_{t=2}^{T} \Lambda_{i} f_{t} e_{i, t-1}^{\prime}-\frac{1}{T} \sum_{t=2}^{T} \Lambda_{i} f_{t} \bar{e}_{i}^{\prime} \\
& \stackrel{p}{\longrightarrow} 0-0,
\end{aligned}
$$

as $T \rightarrow \infty$.

Finally,

$$
\begin{aligned}
\frac{1}{T} \sum_{t=2}^{T} \Delta E_{i, t} \tilde{F}_{t-1}^{\prime} \Lambda_{i}^{\prime} & =\frac{1}{T} \sum_{t=2}^{T}\left(e_{i, t}-e_{i, t-1}\right) \tilde{F}_{t-1}^{\prime} \Lambda_{i}^{\prime} \\
& =\frac{1}{T} e_{i, T} \tilde{F}_{T-1}^{\prime} \Lambda^{\prime}-\frac{1}{T} e_{i, 1} \tilde{F}_{1}^{\prime} \Lambda^{\prime}-\frac{1}{T} \sum_{t=2}^{T} e_{i, t-1} f_{t-1}^{\prime} \Lambda_{i}^{\prime} \\
& \stackrel{p}{\longrightarrow} 0-0-0,
\end{aligned}
$$

as $T \rightarrow \infty$. Hence,

$$
\frac{1}{N} \sum_{i=1}^{N} \frac{1}{T} \sum_{t=2}^{T} \Delta Z_{i, t} \tilde{Z}_{i, t-1}^{\prime} \stackrel{\mathcal{L}, p}{\longrightarrow} \int \mathbf{d} B_{F \Lambda} \tilde{B}_{F \Lambda}+\Theta_{F \Lambda}+\gamma_{1}-\Upsilon,
$$

as $T \rightarrow \infty$ followed by $N \rightarrow \infty$.

If the idiosyncratic components are I(1) and their true DGP includes (4), such that $\Delta E_{i, t}=e_{i, t}$ and $\tilde{E}_{i, t-1}=\tilde{S}_{i, t-1}$, using Lemmas 1 (c), 2 (c) and 3 (d) and (f), we obtain

$$
\frac{1}{N} \sum_{i=1}^{N} \frac{1}{T} \sum_{t=2}^{T} \Delta Z_{i, t} \tilde{Z}_{i, t-1}^{\prime} \stackrel{\mathcal{L}, p}{\longrightarrow} \int \mathbf{d} B_{F \Lambda} \tilde{B}_{F \Lambda}+\Theta_{F \Lambda}-\frac{1}{2} \Psi+\Delta,
$$

as $T \rightarrow \infty$ followed by $N \rightarrow \infty$.

For the denominator in (20) consider

$$
\begin{array}{r}
\sum_{i=1}^{N} \sum_{t=2}^{T} \tilde{Z}_{i, t-1} \tilde{Z}_{i, t-1}^{\prime}=\sum_{i=1}^{N} \sum_{t=2}^{T}\left(\Lambda_{i} \tilde{F}_{t-1}+\tilde{E}_{i, t-1}\right)\left(\Lambda_{i} \tilde{F}_{t-1}+\tilde{E}_{i, t-1}\right)^{\prime} \\
=\sum_{i=1}^{N} \sum_{t=2}^{T}\left(\Lambda_{i} \tilde{F}_{t-1} \tilde{F}_{t-1}^{\prime} \Lambda_{i}^{\prime}+\tilde{E}_{i, t-1} \tilde{E}_{i, t-1}^{\prime}\right. \\
\left.\quad+\Lambda_{i} \tilde{F}_{t-1} \tilde{E}_{i, t-1}^{\prime}+\tilde{E}_{i, t-1} \tilde{F}_{t-1}^{\prime} \Lambda_{i}^{\prime}\right) .
\end{array}
$$


If the idiosyncratic components are given by (3), we have $\left.\sum_{i=1}^{N}\left(O_{p}\left(T^{2}\right)+O_{p}(T)+O_{p}(T)+O_{p}(T)\right)\right)$, in (22). So, $\frac{1}{T^{2}} \sum_{t=2}^{T} \tilde{Z}_{i, t-1} \tilde{Z}_{i, t-1}^{\prime} \Longrightarrow \Lambda_{i} \int \tilde{B}_{F} \tilde{B}_{F}^{\prime} \Lambda_{i}^{\prime}$ as $T \rightarrow \infty$, and $\frac{1}{N} \sum_{i=1}^{N} \Lambda_{i} \int \tilde{B}_{F} \tilde{B}_{F}^{\prime} \Lambda_{i}^{\prime} \stackrel{p}{\longrightarrow}$ $\int \tilde{B}_{F \Lambda} \tilde{B}_{F \Lambda}^{\prime}$ as $N \rightarrow \infty$.

For I(1) idiosyncratic components given by (4), we find using Lemmas 1 (d), 2 (d) and 3(g)

$$
\frac{1}{N} \sum_{i=1}^{N} \frac{1}{T^{2}} \sum_{t=2}^{T} \tilde{Z}_{i, t-1} \tilde{Z}_{i, t-1}^{\prime} \stackrel{\mathcal{L}, p}{\longrightarrow} \int \tilde{B}_{\Lambda} \tilde{B}_{\Lambda}^{\prime}+\frac{1}{6} \Psi
$$

as $T \rightarrow \infty$ followed by $N \rightarrow \infty$. Combining the above given results with those of A (a) or B(a) yields Proposition 1 A (b) and B(b).

\section{B.3 Proposition 1 (c): Divergence of $t_{\tilde{\rho}}$}

The $t$-statistic for $\tilde{\rho}=1$ is given by

$$
t_{\tilde{\rho}}=(\tilde{\rho}-1) s^{-1}\left(\sum_{i=1}^{N} \sum_{t=2}^{T} \tilde{u}_{i, t-1} \tilde{u}_{i, t-1}^{\prime}\right)^{\frac{1}{2}},
$$

where

$$
\begin{aligned}
s^{2} & =\frac{1}{N} \sum_{i=1}^{N} \frac{1}{T} \sum_{t=2}^{T}\left(\Delta \tilde{u}_{i, t}-(\tilde{\rho}-1) \tilde{u}_{i, t-1}\right)^{2} \\
& =\frac{1}{N} \sum_{i=1}^{N} \frac{1}{T} \sum_{t=2}^{T}\left(\Delta \tilde{u}_{i, t}^{2}+0_{p}(1)\right) .
\end{aligned}
$$

As

$$
\frac{1}{N} \sum_{i=1}^{N} \frac{1}{T} \sum_{t=2}^{T} \Delta \tilde{u}_{i, t}^{2}=\frac{1}{N} \sum_{i=1}^{N} \frac{1}{T} \sum_{t=2}^{T}(1-\tilde{\beta}) \Delta Z_{i, t} \Delta \tilde{Z}_{i, t}^{\prime}(1-\tilde{\beta})^{\prime},
$$

which is $O_{p}(1)$ whether the idiosyncratic components are $\mathrm{I}(0)$ or $\mathrm{I}(1), s^{2}$ is $O_{p}(1)$. Furthermore, $T(\tilde{\rho}-1)$ and $\frac{1}{N} \sum_{i=1}^{N} \frac{1}{T^{2}} \sum_{t=2}^{T} \tilde{u}_{i, t-1} \tilde{u}_{i, t-1}^{\prime}$ are $O_{p}(1)$ as well whether $E_{i, t}$ is given by (3) or (4), as shown above. Hence,

$$
\begin{aligned}
t_{\tilde{\rho}} & =\sqrt{N} T(\tilde{\rho}-1) s^{-1}\left(\frac{1}{N} \sum_{i=1}^{N} \frac{1}{T^{2}} \sum_{t=2}^{T} \tilde{u}_{i, t-1} \tilde{u}_{i, t-1}^{\prime}\right)^{\frac{1}{2}} \\
& =\sqrt{N} O_{p}(1),
\end{aligned}
$$

which diverges at rate $\sqrt{N}$ as $T \rightarrow \infty$ followed by $N \rightarrow \infty$.

\section{Proof of Proposition 2}

\section{C.1 Proposition 2 (a): Convergence of $\tilde{\beta}_{i}$}

For each panel unit $i$, the estimator of $\beta_{i}$ is given by $\tilde{\beta}_{i}=\left(\sum_{t=1}^{T} \tilde{Y}_{i, t} \tilde{X}_{i, t}^{\prime}\right)\left(\sum_{t=1}^{T} \tilde{X}_{i, t} \tilde{X}_{i, t}^{\prime}\right)^{-1}$. Consider the numerator

$$
\begin{aligned}
\sum_{t=1}^{T} \tilde{Y}_{i, t} \tilde{X}_{i, t}^{\prime} & =\sum_{t=1}^{T}\left(\lambda_{1 i}^{\prime} \tilde{F}_{t}^{Y}+\tilde{E}_{i, t}^{Y}\right)\left(\lambda_{2 i}^{\prime} \tilde{F}_{t}^{X}+\tilde{E}_{i, t}^{X}\right)^{\prime} \\
& =\sum_{t=1}^{T}\left(\lambda_{1 i}^{\prime} \tilde{F}_{t}^{Y} \tilde{F}_{t}^{X \prime} \lambda_{21}+\tilde{E}_{i, t}^{Y} \tilde{E}_{i, t}^{X \prime}+\lambda_{1 i}^{\prime} \tilde{F}_{t}^{Y} \tilde{E}_{i, t}^{X \prime}+\tilde{E}_{i, t}^{Y} \tilde{F}_{t}^{X \prime} \lambda_{21}\right) .
\end{aligned}
$$

If the idiosyncratic term is given by (3), we have $O_{p}\left(T^{2}\right)+O_{p}(T)+O_{p}(T)+O_{p}(T)$ in (23). So, 
as $T \rightarrow \infty, \frac{1}{T^{2}} \sum_{t=1}^{T} \tilde{Y}_{i, t} \tilde{X}_{i, t}^{\prime} \Longrightarrow \lambda_{1 i}^{\prime} \int \tilde{B}_{F}^{Y} \tilde{B}_{F}^{X \prime} \lambda_{2 i}$ from the first result of Lemma 1 (d).

If the idiosyncratic terms are also I(1), such that the DGP includes (4), all terms in (23) are $O_{p}\left(T^{2}\right)$ when summed over $T$. Using Lemmas 1 (d), $2(\mathrm{~d})$ and $3(\mathrm{~g})$ we find as $T \rightarrow \infty$,

$$
\frac{1}{T^{2}} \sum_{t=1}^{T} \tilde{Y}_{i, t} \tilde{X}_{i, t}^{\prime} \Longrightarrow\left(\lambda_{1 i}^{\prime} \int \tilde{B}_{F}^{Y} \tilde{B}_{F}^{X \prime} \lambda_{2 i}+\int \tilde{B}_{i}^{Y} \tilde{B}_{i}^{X \prime}+\lambda_{1 i}^{\prime} \int \tilde{B}_{F}^{Y} \tilde{B}_{i}^{X \prime}+\int \tilde{B}_{i}^{Y} \tilde{B}_{F}^{X \prime} \lambda_{21}\right) .
$$

Now the denominator of $\tilde{\beta}_{i}$ is given by

$$
\begin{aligned}
\sum_{t=1}^{T} \tilde{X}_{i, t} \tilde{X}_{i, t}^{\prime} & =\sum_{t=1}^{T}\left(\lambda_{1 i}^{\prime} \tilde{F}_{t}^{X}+\tilde{E}_{i, t}^{X}\right)\left(\lambda_{2 i}^{\prime} \tilde{F}_{t}^{X}+\tilde{E}_{i, t}^{X}\right)^{\prime} \\
& =\sum_{t=1}^{T}\left(\lambda_{2 i}^{\prime} \tilde{F}_{t}^{X} \tilde{F}_{t}^{X \prime} \lambda_{21}+\tilde{E}_{i, t}^{X} \tilde{E}_{i, t}^{X \prime}+\lambda_{2 i}^{\prime} \tilde{F}_{t}^{X} \tilde{E}_{i, t}^{X \prime}+\tilde{E}_{i, t}^{X} \tilde{F}_{t}^{X \prime} \lambda_{21}\right) .
\end{aligned}
$$

Similar to the results for the numerator, the terms in $(24)$ are $O_{p}\left(T^{2}\right)+O_{p}(T)+O_{p}(T)+O_{p}(T)$, if the DGP contains (3). Hence, $\frac{1}{T^{2}} \sum_{t=1}^{T} \tilde{X}_{i, t} \tilde{X}_{i, t}^{\prime} \Longrightarrow \lambda_{2 i}^{\prime} \int \tilde{B}_{F}^{X} \tilde{B}_{F}^{X \prime} \lambda_{2 i}$ as $T \rightarrow \infty$.

If the true DGP contains (4), all terms in (24) are $O_{p}\left(T^{2}\right)$ and we have

$$
\frac{1}{T^{2}} \sum_{t=1}^{T} \tilde{X}_{i, t} \tilde{X}_{i, t}^{\prime} \Longrightarrow\left(\lambda_{2 i}^{\prime} \int \tilde{B}_{F}^{X} \tilde{B}_{F}^{X \prime} \lambda_{2 i}+\int \tilde{B}_{i}^{X} \tilde{B}_{i}^{X \prime}+\lambda_{2 i}^{\prime} \int \tilde{B}_{F}^{X} \tilde{B}_{i}^{X \prime}+\int \tilde{B}_{i}^{X} \tilde{B}_{F}^{X \prime} \lambda_{21}\right),
$$

as $T \rightarrow \infty$.

Combining the results given above yields Proposition 2 A(a) and B(a).

\section{C.2 Proposition 2 (b): Convergence of $Z_{\tilde{\rho}_{N T}-1}$ and $\tilde{Z}_{\tilde{\rho}_{N T}-1}$}

The residuals from the individual first stage regression are given by $\tilde{u}_{i, t}=\left(1,-\tilde{\beta}_{i}\right) Z_{i, t}=Y_{i, t}-\tilde{\beta}_{i} X_{i, t}$. Consider first

$$
\sum_{t=2}^{T} \Delta \tilde{u}_{i, t} \tilde{u}_{i, t-1}=\sum_{t=2}^{T}\left(1,-\tilde{\beta}_{i}\right) \Delta Z_{i, t} \tilde{Z}_{i, t-1}^{\prime}\left(1,-\tilde{\beta}_{i}\right)^{\prime} .
$$

Now,

$$
\begin{aligned}
\sum_{t=2}^{T} \Delta Z_{i, t} \tilde{Z}_{i, t-1}^{\prime} & =\sum_{t=2}^{T}\left(\Lambda_{i} f_{t}+\Delta E_{i, t}\right)\left(\Lambda_{i} \tilde{F}_{t-1}+\tilde{E}_{i, t-1}\right)^{\prime} \\
& =\sum_{t=2}^{T}\left(\Lambda_{i} f_{t} \tilde{F}_{t-1}^{\prime} \Lambda_{i}^{\prime}+\Delta E_{i, t} \tilde{E}_{i, t-1}^{\prime}+\Lambda_{i} f_{t} \tilde{E}_{i, t-1}^{\prime}+\Delta E_{i, t} \tilde{F}_{t-1}^{\prime} \Lambda_{i}^{\prime}\right) .
\end{aligned}
$$

From Lemma 1 (c), $\frac{1}{T} \sum_{t=2}^{T} \Lambda_{i} f_{t} \tilde{F}_{t-1} \Lambda_{i}^{\prime} \Longrightarrow \int \Lambda_{i}\left(\mathbf{d} B_{F} \tilde{B}_{F}+\Theta\right) \Lambda_{i}^{\prime}$ as $T \rightarrow \infty$. If the idiosyncratic terms are $\mathrm{I}(0)$, i.e. the true DGP is given by (3),

$$
\sum_{t=2}^{T} \Delta E_{i, t} \tilde{E}_{i, t-1}^{\prime}=\sum_{t=2}^{T}\left(\left(e_{i, t}-e_{i, t-1}\right) e_{i, t-1}^{\prime}-\left(e_{i, t}-e_{i, t-1}\right) \bar{e}_{i}\right),
$$

where $\bar{e}_{i}=\frac{1}{T} \sum_{t=1}^{T} e_{i, t}$. Now, $\frac{1}{T} \sum_{t=2}^{T} e_{i, t} e_{i, t-1}^{\prime} \stackrel{p}{\longrightarrow} \gamma_{i 1}$ as $T \rightarrow \infty$, with $\gamma_{i 1}=\lim _{T \rightarrow \infty} \frac{1}{T} \sum_{i=1}^{T} \mathrm{E}\left(e_{i, t} e_{i, t-1}\right)$. Also, $\frac{1}{T} \sum_{t=2}^{T} e_{i, t-1} e_{i, t-1}^{\prime} \stackrel{p}{\longrightarrow} \Upsilon_{i}$ as $T \rightarrow \infty$. Furthermore, $\frac{1}{T} \sum_{t=2}^{T} e_{i, t} \bar{e}_{i}^{\prime} \stackrel{p}{\longrightarrow} 0$ and $\frac{1}{T} \sum_{t=2}^{T} e_{i, t-1} \bar{e}_{i}^{\prime} \stackrel{p}{\longrightarrow}$ 0 as $T \rightarrow \infty$. Hence, $\frac{1}{T} \sum_{t=2}^{T} \Delta E_{i, t} \tilde{E}_{i, t-1}^{\prime} \stackrel{p}{\longrightarrow} \gamma_{i 1}-\Upsilon_{i}$ as $T \rightarrow \infty$. 
For the third term in (26) we have,

$$
\begin{aligned}
\frac{1}{T} \sum_{t=2}^{T} \Lambda_{i} f_{t} \tilde{E}_{i, t-1}^{\prime} & =\frac{1}{T} \sum_{t=2}^{T} \Lambda_{i} f_{t}\left(e_{i, t-1}-\bar{e}_{i}\right)^{\prime} \\
& =\frac{1}{T} \sum_{t=2}^{T} \Lambda_{i} f_{t} e_{i, t-1}^{\prime}-\frac{1}{T} \sum_{t=2}^{T} \Lambda_{i} f_{t} \bar{e}_{i}^{\prime} \\
& \stackrel{p}{\longrightarrow} 0-0,
\end{aligned}
$$

as $T \rightarrow \infty$. Finally,

$$
\begin{aligned}
\frac{1}{T} \sum_{t=2}^{T} \Delta E_{i, t} \tilde{F}_{t-1}^{\prime} \Lambda_{i}^{\prime} & =\frac{1}{T} \sum_{t=2}^{T}\left(e_{i, t}-e_{i, t-1}\right) \tilde{F}_{t-1}^{\prime} \Lambda_{i}^{\prime} \\
& =\frac{1}{T} e_{i, T} \tilde{F}_{T-1}^{\prime} \Lambda^{\prime}-\frac{1}{T} e_{i, 1} \tilde{F}_{1}^{\prime} \Lambda^{\prime}-\frac{1}{T} \sum_{t=2}^{T} e_{i, t-1} f_{t-1}^{\prime} \Lambda_{i}^{\prime} \\
& \stackrel{p}{\longrightarrow} 0-0-0,
\end{aligned}
$$

as $T \rightarrow \infty$. Hence,

$$
\frac{1}{T} \sum_{t=2}^{T} \Delta Z_{i, t} \tilde{Z}_{i, t-1}^{\prime} \Longrightarrow \Lambda_{i}\left(\int \mathbf{d} B_{F} \tilde{B}_{F}+\Theta\right) \Lambda_{i}^{\prime}+\gamma_{i 1}-\Upsilon_{i}
$$

as $T \rightarrow \infty$.

If the idiosyncratic components are I(1) and their true DGP includes (4), such that $\Delta E_{i, t}=e_{i, t}$ and $\tilde{E}_{i, t-1}=\tilde{S}_{i, t-1}$, using Lemmas 1 (c), 2 (c) and 3 (d) and (f), we obtain

$$
\frac{1}{T} \sum_{t=2}^{T} \Delta Z_{i, t} \tilde{Z}_{i, t-1}^{\prime} \Longrightarrow\left(\Lambda_{i}^{\prime}\left(\int \mathbf{d} B_{F} \tilde{B}_{F}+\Theta\right) \Lambda_{i}^{\prime}+\int \mathbf{d} B_{i} \tilde{B}_{i}^{\prime}+\Delta_{i}+\Lambda_{i} \int \mathbf{d} B_{F} \tilde{B}_{i}+\int \mathbf{d} B_{i} \tilde{B}_{F}^{\prime} \Lambda_{i}^{\prime}\right),
$$

as $T \rightarrow \infty$.

Furthermore, note that the residuals $\tilde{v}_{i, t}=\Delta \tilde{u}_{i, t}+o_{p}(1)$ regardless of whether they were obtained from the poooled regression (10) or the individual regression (12). Now,

$$
\begin{aligned}
\hat{\boldsymbol{\lambda}}_{i} & =T^{-1} \sum_{s=1}^{J} \omega_{s J} \sum_{t=s+1}^{T} \tilde{v}_{i, t} \tilde{v}_{i, t-s} \\
& =T^{-1} \sum_{s=1}^{J} \omega_{s J} \sum_{t=s+1}^{T} \Delta \tilde{u}_{i, t} \Delta \tilde{u}_{i, t-s}+o_{p}(1) \\
& =T^{-1} \sum_{s=1}^{J} \omega_{s J} \sum_{t=s+1}^{T}\left(1,-\tilde{\beta}_{i}\right) \Delta \tilde{Z}_{i, t} \Delta \tilde{Z}_{i, t-s}^{\prime}\left(1,-\tilde{\beta}_{i}\right)^{\prime}+o_{p}(1) .
\end{aligned}
$$

Expanding $\Delta \tilde{Z}_{i, t} \Delta \tilde{Z}_{i, t-s}^{\prime}$ in terms of the common factors and unobserved components we obtain the following four terms and convergence results for suitable choices of bandwidth $J$ and kernel function $\omega_{s J}$. First,

$$
T^{-1} \sum_{s=1}^{J} \omega_{s J} \sum_{t=s+1}^{T} \Lambda_{i} \tilde{f}_{i, t} \tilde{f}_{i, t-s}^{\prime} \Lambda_{i}^{\prime} \stackrel{p}{\longrightarrow} \Lambda_{i} \Omega \Lambda_{i}^{\prime}
$$

Next,

$$
T^{-1} \sum_{s=1}^{J} \omega_{s J} \sum_{t=s+1}^{T} \Lambda_{i} \tilde{f}_{i, t} \Delta \tilde{E}_{i, t-s}^{\prime} \stackrel{p}{\longrightarrow} 0
$$


and

$$
T^{-1} \sum_{s=1}^{J} \omega_{s J} \sum_{t=s+1}^{T} \Delta \tilde{E}_{i, t} \tilde{f}_{i, t-s}^{\prime} \Lambda_{i} \stackrel{p}{\longrightarrow} 0
$$

due to the independence of common factors and idiosyncratic components. Finally,

$$
T^{-1} \sum_{s=1}^{J} \omega_{s J} \sum_{t=s+1}^{T} \Delta \tilde{E}_{i, t} \Delta \tilde{E}_{i, t-s}^{\prime} \stackrel{p}{\longrightarrow} \lim _{T \rightarrow \infty} \frac{1}{T} \sum_{t=1}^{T} \mathrm{E}\left(e_{i, t} \tilde{E}_{i, t}\right),
$$

which is $\gamma_{1 i}-\Upsilon_{i}$ if the idiosyncratic components are stationary, and $\Delta_{i}$ if they are $\mathrm{I}(1)$.

Now consider

$$
\sum_{t=2}^{T} \Delta \tilde{u}_{i, t} \tilde{u}_{i, t-1}=\sum_{t=2}^{T}\left(1,-\tilde{\beta}_{i}\right) \Delta Z_{i, t} \tilde{Z}_{i, t-1}^{\prime}\left(1,-\tilde{\beta}_{i}\right)^{\prime}
$$

We have

$$
\begin{array}{r}
\sum_{t=2}^{T} \tilde{Z}_{i, t-1} \tilde{Z}_{i, t-1}^{\prime}= \\
=\sum_{t=2}^{T}\left(\Lambda_{i} \tilde{F}_{t-1}+\tilde{E}_{i, t-1}\right)\left(\Lambda_{i} \tilde{F}_{t-1}+\tilde{E}_{i, t-1}\right)^{\prime} \\
=\sum_{t=2}^{T}\left(\Lambda_{i} \tilde{F}_{t-1} \tilde{F}_{t-1}^{\prime} \Lambda_{i}^{\prime}+\tilde{E}_{i, t-1} \tilde{E}_{i, t-1}^{\prime}\right. \\
\left.\quad+\Lambda_{i} \tilde{F}_{t-1} \tilde{E}_{i, t-1}^{\prime}+\tilde{E}_{i, t-1} \tilde{F}_{t-1}^{\prime} \Lambda_{i}^{\prime}\right) .
\end{array}
$$

If the idiosyncratic components are given by (3), when summed over $T$ the first term in (32) is $O_{p}\left(T^{2}\right)$, while the remaining three are $O_{p}(T)$. So, $\frac{1}{T^{2}} \sum_{t=2}^{T} \tilde{Z}_{i, t-1} \tilde{Z}_{i, t-1}^{\prime} \Longrightarrow \Lambda_{i} \int \tilde{B}_{F} \tilde{B}_{F}^{\prime} \Lambda_{i}^{\prime}$ as $T \rightarrow \infty$.

For I(1) idiosyncratic components given by (4), we find using Lemmas 1 (d), 2 (d) and 3(g)

$$
\frac{1}{T^{2}} \sum_{t=2}^{T} \tilde{Z}_{i, t-1} \tilde{Z}_{i, t-1}^{\prime} \Longrightarrow\left(\Lambda_{i} \int \tilde{B}_{F} \tilde{B}_{F}^{\prime} \Lambda_{i}^{\prime}+\int \tilde{B}_{i} \tilde{B}_{i}^{\prime}+\Lambda_{i} \int \tilde{B}_{F} \tilde{B}_{i}^{\prime}+\int \tilde{B}_{i} \tilde{B}_{F}^{\prime} \Lambda_{i}^{\prime}\right)
$$

as $T \rightarrow \infty$.

Next, we partition the long-run covariance matrix of the common non-stationary factors $\Omega$ conformable to the partition of $B_{F}=\left(B_{F}^{Y^{\prime}}, B_{F}^{X \prime}\right)^{\prime}$, such that

$$
\Omega=\left[\begin{array}{ll}
\Omega_{11} & \Omega_{21}^{\prime} \\
\Omega_{21} & \Omega_{22}
\end{array}\right] .
$$

We use the block-triangular decomposition $\Omega=L^{\prime} L$, with

$$
L=\left[\begin{array}{cc}
L_{11} & 0 \\
L_{21} & L_{22}
\end{array}\right]
$$

where $L_{11}=\left(\Omega_{11}-\Omega_{21}^{\prime} \Omega_{22}^{-1} \Omega_{21}\right)^{\frac{1}{2}}, L_{21}=\Omega_{22}^{-\frac{1}{2}} \Omega_{21}$, and $L_{22}=\Omega_{22}^{\frac{1}{2}}$. Note that $\Omega_{22}>0$ by Assumption 1.

Now, $\tilde{B}_{F}=L^{\prime} \tilde{W}_{F}$, where $\tilde{W}_{F}$ is a demeaned $k$-vector standard Brownian motion. Furthermore, denote $\boldsymbol{\eta}_{i}^{\prime}=\left(1,-\tilde{\boldsymbol{b}}_{i A}\right)$, and $\left.\boldsymbol{\kappa}^{\prime}=\left(\boldsymbol{I}_{k_{Y}},-\left(\int \tilde{W}_{F}^{Y} \tilde{W}_{F}^{X \prime}\right)\left(\int \tilde{W}_{F}^{X} \tilde{W}_{F}^{X \prime}\right)^{-1}\right)\right)$. Then, $L \Lambda_{i}^{\prime} \boldsymbol{\eta}_{i}=\boldsymbol{\kappa} L_{11} \lambda_{1 i}$, and $\boldsymbol{\eta}_{i}^{\prime} \tilde{B}_{F}=\lambda_{1 i}^{\prime} L_{11}^{\prime} \tilde{Q}_{F}$, with $\tilde{Q}_{F}=\tilde{W}_{F}^{Y}-\left(\int \tilde{W}_{F}^{Y} \tilde{W}_{F}^{X \prime}\right)\left(\int \tilde{W}_{F}^{X} \tilde{W}_{F}^{X \prime}\right)^{-1} \tilde{W}_{F}^{X}$. Finally,

$$
\boldsymbol{\eta}_{i}^{\prime} \int \mathbf{d} B_{F} \tilde{B}_{F}^{\prime} \boldsymbol{\eta}_{i}=\lambda_{1 i}^{\prime} L_{11}^{\prime} \int \mathbf{d} Q_{F} \tilde{Q}_{F}^{\prime} L_{11} \lambda_{1 i},
$$

and

$$
\boldsymbol{\eta}_{i}^{\prime} \int \tilde{B}_{F} \tilde{B}_{F}^{\prime} \boldsymbol{\eta}_{i}=\lambda_{1 i}^{\prime} L_{11}^{\prime} \int \tilde{Q}_{F} \tilde{Q}_{F}^{\prime} L_{11} \lambda_{1 i}
$$


Combining the above given results with those of A (a) or B (a) yields the convergence results for $Z_{\tilde{\rho}_{N T}-1}$ and $\tilde{Z}_{\tilde{\rho}_{N T}-1}$. 


\section{Tables}

Table 1: Cross-member cointegration without serial correlation.

\begin{tabular}{|c|c|c|c|c|c|c|c|c|c|}
\hline$\overline{\mathrm{N}}$ & 25 & 50 & 100 & 25 & 50 & 100 & 25 & 50 & 100 \\
\hline $\mathrm{T}$ & \multicolumn{3}{|c|}{ Kao $D F_{\rho}$} & \multicolumn{3}{|c|}{ Kao $D F_{t}$} & \multicolumn{3}{|c|}{ Pedroni Panel $-\rho$} \\
\hline 50 & 0.80 & 0.83 & 0.85 & 0.27 & 0.27 & 0.32 & 0.90 & 0.91 & 0.92 \\
\hline 100 & 0.81 & 0.85 & 0.87 & 0.26 & 0.30 & 0.33 & 0.90 & 0.93 & 0.93 \\
\hline 250 & 0.83 & 0.89 & 0.88 & 0.28 & 0.34 & 0.37 & 0.92 & 0.94 & 0.95 \\
\hline $\mathrm{T}$ & \multicolumn{3}{|c|}{ Pedroni Panel $-t$} & \multicolumn{3}{|c|}{ Pedroni Group $-\rho$} & \multicolumn{3}{|c|}{ Pedroni Group $-t$} \\
\hline 50 & 0.93 & 0.93 & 0.93 & 0.77 & 0.78 & 0.83 & 0.86 & 0.85 & 0.88 \\
\hline 100 & 0.92 & 0.93 & 0.93 & 0.80 & 0.84 & 0.84 & 0.85 & 0.87 & 0.86 \\
\hline 250 & 0.94 & 0.94 & 0.95 & 0.84 & 0.85 & 0.88 & 0.87 & 0.88 & 0.91 \\
\hline $\mathrm{T}$ & \multicolumn{3}{|c|}{ Idiosyncratic Panel - $\rho$} & \multicolumn{3}{|c|}{ Idiosyncratic Group $-\rho$} & \multicolumn{3}{|c|}{ Aznar/Johansen } \\
\hline 50 & 1.00 & 1.00 & 1.00 & 1.00 & 1.00 & 1.00 & 0.08 & 0.08 & 0.06 \\
\hline 100 & 1.00 & 1.00 & 1.00 & 1.00 & 1.00 & 1.00 & 0.04 & 0.04 & 0.05 \\
\hline 250 & 1.00 & 1.00 & 1.00 & 1.00 & 1.00 & 1.00 & 0.05 & 0.05 & 0.05 \\
\hline
\end{tabular}


Table 2: I(1) common factors and I(1) idiosyncratic components without serial correlation.

\begin{tabular}{|c|c|c|c|c|c|c|c|c|c|}
\hline $\mathrm{N}$ & 25 & 50 & 100 & 25 & 50 & 100 & 25 & 50 & 100 \\
\hline $\mathrm{T}$ & \multicolumn{3}{|c|}{ Као $D F_{\rho}$} & \multicolumn{3}{|c|}{ Kao $D F_{t}$} & \multicolumn{3}{|c|}{ Pedroni Panel $-\rho$} \\
\hline 50 & 0.00 & 0.00 & 0.00 & 0.00 & 0.00 & 0.00 & 0.17 & 0.23 & 0.33 \\
\hline 100 & 0.00 & 0.00 & 0.00 & 0.00 & 0.00 & 0.00 & 0.19 & 0.31 & 0.36 \\
\hline 250 & 0.00 & 0.00 & 0.00 & 0.00 & 0.00 & 0.00 & 0.25 & 0.29 & 0.38 \\
\hline $\mathrm{T}$ & \multicolumn{3}{|c|}{ Pedroni Panel $-t$} & \multicolumn{3}{|c|}{ Pedroni Group $-\rho$} & \multicolumn{3}{|c|}{ Pedroni Group $-t$} \\
\hline 50 & 0.46 & 0.51 & 0.57 & 0.10 & 0.12 & 0.18 & 0.34 & 0.42 & 0.49 \\
\hline 100 & 0.44 & 0.49 & 0.58 & 0.13 & 0.21 & 0.24 & 0.30 & 0.40 & 0.45 \\
\hline 250 & 0.44 & 0.48 & 0.54 & 0.19 & 0.21 & 0.33 & 0.30 & 0.33 & 0.43 \\
\hline $\mathrm{T}$ & \multicolumn{3}{|c|}{ Idiosyncratic Panel $-\rho$} & \multicolumn{3}{|c|}{ Idiosyncratic Group $-\rho$} & \multicolumn{3}{|c|}{ Aznar/Johansen } \\
\hline 50 & 0.04 & 0.04 & 0.12 & 0.01 & 0.02 & 0.01 & 0.08 & 0.08 & 0.06 \\
\hline 100 & 0.06 & 0.05 & 0.05 & 0.02 & 0.02 & 0.01 & 0.04 & 0.04 & 0.05 \\
\hline 250 & 0.07 & 0.06 & 0.04 & 0.06 & 0.03 & 0.03 & 0.06 & 0.04 & 0.05 \\
\hline
\end{tabular}


Table 3: Cross-member cointegration with MA(1) errors

\begin{tabular}{|c|c|c|c|c|c|c|c|c|c|}
\hline$\overline{\mathrm{N}}$ & 25 & 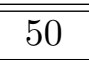 & 100 & 25 & $\overline{50}$ & 100 & 25 & 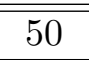 & 100 \\
\hline $\mathrm{T}$ & \multicolumn{3}{|c|}{$\mathrm{Kao}-\rho^{*}$} & \multicolumn{3}{|c|}{ Kao - ADF } & \multicolumn{3}{|c|}{ Pedroni - Panel $-\rho$} \\
\hline 25 & 0.27 & 0.32 & 0.35 & 0.48 & 0.50 & 0.54 & 0.68 & 0.90 & 0.88 \\
\hline 50 & 0.39 & 0.47 & 0.49 & 0.54 & 0.62 & 0.62 & 0.84 & 0.96 & 0.95 \\
\hline 100 & 0.52 & 0.54 & 0.55 & 0.64 & 0.67 & 0.69 & 0.93 & 1.00 & 0.96 \\
\hline $\mathrm{T}$ & \multicolumn{3}{|c|}{ Pedroni - Panel - $t$} & \multicolumn{3}{|c|}{ Pedroni - Group - $\rho$} & \multicolumn{3}{|c|}{ Pedroni - Group - $t$} \\
\hline 25 & 0.76 & 0.92 & 0.91 & 0.33 & 0.67 & 0.62 & 0.52 & 0.79 & 0.77 \\
\hline 50 & 0.83 & 0.96 & 0.94 & 0.665 & 0.94 & 0.89 & 0.67 & 0.89 & 0.85 \\
\hline 100 & 0.92 & 0.99 & 0.95 & 0.88 & 1.00 & 0.94 & 0.78 & 0.95 & 0.88 \\
\hline $\mathrm{T}$ & \multicolumn{3}{|c|}{ Idiosyncratic- Panel - $t$} & \multicolumn{3}{|c|}{ Idiosyncratic - Group - $\rho$} & \multicolumn{3}{|c|}{ Aznar/Johansen } \\
\hline 25 & 1.00 & 1.00 & 1.00 & 1.00 & 1.00 & 1.00 & 0.12 & 0.12 & 0.11 \\
\hline 50 & 1.00 & 1.00 & 1.00 & 1.00 & 1.00 & 1.00 & 0.09 & 0.11 & 0.09 \\
\hline 100 & 1.00 & 1.00 & 1.00 & 1.00 & 1.00 & 1.00 & 0.08 & 0.10 & 0.08 \\
\hline
\end{tabular}

Rejection frequencies are based on $5 \%$ asymptotic critical values. 
Table 4: I(1) common factors and I(1) idiosyncratic components with MA(1) errors

\begin{tabular}{|c|c|c|c|c|c|c|c|c|c|}
\hline$\overline{\mathrm{N}}$ & 25 & 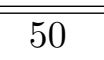 & 100 & 25 & $\overline{50}$ & $\overline{c 100}$ & 25 & $\overline{50}$ & 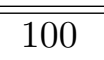 \\
\hline $\mathrm{T}$ & \multicolumn{3}{|c|}{$\mathrm{KaO}-\rho^{*}$} & \multicolumn{3}{|c|}{$\mathrm{Kao}-\mathrm{ADF}$} & \multicolumn{3}{|c|}{ Pedroni - Panel - $\rho$} \\
\hline 25 & 0.17 & 0.17 & 0.23 & 0.59 & 0.65 & 0.69 & 0.00 & 0.00 & 0.00 \\
\hline 50 & 0.23 & 0.28 & 0.36 & 0.63 & 0.74 & 0.75 & 0.02 & 0.02 & 0.03 \\
\hline 100 & 0.34 & 0.39 & 0.45 & 0.74 & 0.81 & 0.80 & 0.10 & 0.08 & 0.14 \\
\hline $\mathrm{T}$ & \multicolumn{3}{|c|}{ Pedroni - Panel $-t$} & \multicolumn{3}{|c|}{ Pedroni - Group - $\rho$} & \multicolumn{3}{|c|}{ Pedroni - Group - $t$} \\
\hline 25 & 0.03 & 0.02 & 0.04 & 0.00 & 0.00 & 0.00 & 0.03 & 0.02 & 0.04 \\
\hline 50 & 0.06 & 0.04 & 0.08 & 0.00 & 0.00 & 0.01 & 0.04 & 0.03 & 0.06 \\
\hline 100 & 0.13 & 0.10 & 0.18 & 0.02 & 0.01 & 0.04 & 0.07 & 0.05 & 0.10 \\
\hline $\mathrm{T}$ & \multicolumn{3}{|c|}{ Idiosyncratic- Panel - $t$} & \multicolumn{3}{|c|}{ Idiosyncratic - Group - $\rho$} & \multicolumn{3}{|c|}{ Aznar/Johansen } \\
\hline 25 & 0.00 & 0.00 & 0.00 & 0.00 & 0.00 & 0.00 & 0.12 & 0.14 & 0.12 \\
\hline 50 & 0.00 & 0.00 & 0.00 & 0.00 & 0.00 & 0.00 & 0.10 & 0.12 & 0.09 \\
\hline 100 & 0.02 & 0.01 & 0.00 & 0.00 & 0.00 & 0.00 & 0.11 & 0.10 & 0.09 \\
\hline
\end{tabular}

Rejection frequencies are based on $5 \%$ asymptotic critical values. 
Table 5: Cointegration in $F_{t}$ but not in $E_{i, t}$ without serial correlation.

\begin{tabular}{|c|c|c|c|c|c|c|c|c|c|}
\hline $\mathrm{N}$ & 25 & 50 & 100 & 25 & 50 & 100 & 25 & 50 & 100 \\
\hline $\mathrm{T}$ & \multicolumn{3}{|c|}{ Kao $D F_{\rho}$} & \multicolumn{3}{|c|}{ Kao $D F_{t}$} & \multicolumn{3}{|c|}{ Pedroni Panel $-\rho$} \\
\hline 50 & 0.00 & 0.00 & 0.00 & 0.00 & 0.00 & 0.00 & 0.45 & 0.59 & 0.73 \\
\hline 100 & 0.00 & 0.00 & 0.00 & 0.00 & 0.00 & 0.00 & 0.60 & 0.79 & 0.85 \\
\hline 250 & 0.00 & 0.00 & 0.00 & 0.00 & 0.00 & 0.00 & 0.70 & 0.85 & 0.93 \\
\hline $\mathrm{T}$ & \multicolumn{3}{|c|}{ Pedroni Panel $-t$} & \multicolumn{3}{|c|}{ Pedroni Group $-\rho$} & \multicolumn{3}{|c|}{ Pedroni Group $-t$} \\
\hline 50 & 0.74 & 0.82 & 0.92 & 0.38 & 0.51 & 0.65 & 0.70 & 0.82 & 0.92 \\
\hline 100 & 0.79 & 0.91 & 0.93 & 0.59 & 0.80 & 0.88 & 0.72 & 0.89 & 0.93 \\
\hline 250 & 0.83 & 0.92 & 0.96 & 0.78 & 0.89 & 0.96 & 0.77 & 0.90 & 0.94 \\
\hline $\mathrm{T}$ & \multicolumn{3}{|c|}{ Idiosyncratic Panel - $\rho$} & \multicolumn{3}{|c|}{ Idiosyncratic Group $-\rho$} & \multicolumn{3}{|c|}{ Aznar/Johansen } \\
\hline 50 & 0.04 & 0.04 & 0.01 & 0.01 & 0.02 & 0.01 & 0.73 & 0.83 & 0.87 \\
\hline 100 & 0.06 & 0.05 & 0.04 & 0.02 & 0.02 & 0.01 & 0.82 & 0.87 & 0.92 \\
\hline 250 & 0.08 & 0.06 & 0.04 & 0.06 & 0.03 & 0.03 & 0.68 & 0.83 & 0.90 \\
\hline
\end{tabular}


Table 6: No cointegration in $F_{t}$ but cointegration in $E_{i, t}$ without serial correlation.

\begin{tabular}{|c|c|c|c|c|c|c|c|c|c|}
\hline$\overline{\mathrm{N}}$ & 25 & 50 & 100 & 25 & 50 & 100 & 25 & $\overline{50}$ & 100 \\
\hline $\mathrm{T}$ & \multicolumn{3}{|c|}{ Kao $D F_{\rho}$} & \multicolumn{3}{|c|}{ Kao $D F_{t}$} & \multicolumn{3}{|c|}{ Pedroni Panel $-\rho$} \\
\hline 50 & 0.16 & 0.18 & 0.17 & 0.02 & 0.04 & 0.03 & 0.58 & 0.60 & 0.64 \\
\hline 100 & 0.20 & 0.24 & 0.25 & 0.02 & 0.04 & 0.05 & 0.60 & 0.66 & 0.69 \\
\hline 250 & 0.25 & 0.30 & 0.32 & 0.03 & 0.04 & 0.07 & 0.61 & 0.70 & 0.75 \\
\hline $\mathrm{T}$ & \multicolumn{3}{|c|}{ Pedroni Panel $-t$} & \multicolumn{3}{|c|}{ Pedroni Group $-\rho$} & \multicolumn{3}{|c|}{ Pedroni Group $-t$} \\
\hline 50 & 0.72 & 0.73 & 0.74 & 0.39 & 0.39 & 0.46 & 0.55 & 0.57 & 0.61 \\
\hline 100 & 0.70 & 0.73 & 0.77 & 0.43 & 0.50 & 0.53 & 0.54 & 0.60 & 0.63 \\
\hline 250 & 0.70 & 0.78 & 0.78 & 0.47 & 0.52 & 0.57 & 0.52 & 0.58 & 0.64 \\
\hline $\mathrm{T}$ & \multicolumn{3}{|c|}{ Idiosyncratic Panel $-\rho$} & \multicolumn{3}{|c|}{ Idiosyncratic Group $-\rho$} & \multicolumn{3}{|c|}{ Aznar/Johansen } \\
\hline 50 & 1.00 & 1.00 & 1.00 & 1.00 & 1.00 & 1.00 & 0.07 & 0.08 & 0.07 \\
\hline 100 & 1.00 & 1.00 & 1.00 & 1.00 & 1.00 & 1.00 & 0.05 & 0.03 & 0.05 \\
\hline 250 & 1.00 & 1.00 & 1.00 & 1.00 & 1.00 & 1.00 & 0.05 & 0.05 & 0.05 \\
\hline
\end{tabular}

$\overline{F_{t}=\sum_{s=1}^{t} \eta_{s}, \text { where } \eta_{t} \sim \operatorname{iidN}\left(0, I_{2}\right), E_{i, t}^{Y}=\sum_{s=1}^{T} \varepsilon_{i, s}^{Y}, \Delta E_{i, t}^{X}=\Delta E_{i, t}^{Y}+\varepsilon_{i, s}^{X},}$ where $\varepsilon_{i, t}=\left(\varepsilon_{i, t}^{Y}, \varepsilon_{i, t}^{X}\right)^{\prime} \sim i d N\left(0, I_{2}\right)$ and $\Lambda_{i}=I_{2}$ for all $i$.

Rejection frequencies are based on $5 \%$ asymptotic critical values. 
Table 7: Cointegration in $F_{t}$ and $E_{i, t}$ with common cointegrating vector without serial correlation.

\begin{tabular}{|c|c|c|c|c|c|c|c|c|c|}
\hline $\mathrm{N}$ & 25 & 50 & 100 & 25 & 50 & 100 & 25 & 50 & 100 \\
\hline $\mathrm{T}$ & \multicolumn{3}{|c|}{ Kao $D F_{\rho}$} & \multicolumn{3}{|c|}{ Kao $D F_{t}$} & \multicolumn{3}{|c|}{ Pedroni Panel $-\rho$} \\
\hline 50 & 1.00 & 1.00 & 1.00 & 1.00 & 1.00 & 1.00 & 1.00 & 1.00 & 1.00 \\
\hline 100 & 1.00 & 1.00 & 1.00 & 1.00 & 1.00 & 1.00 & 1.00 & 1.00 & 1.00 \\
\hline 250 & 1.00 & 1.00 & 1.00 & 1.00 & 1.00 & 1.00 & 1.00 & 1.00 & 1.00 \\
\hline $\mathrm{T}$ & \multicolumn{3}{|c|}{ Pedroni Panel $-t$} & \multicolumn{3}{|c|}{ Pedroni Group $-\rho$} & \multicolumn{3}{|c|}{ Pedroni Group $-t$} \\
\hline 50 & 1.00 & 1.00 & 1.00 & 1.00 & 1.00 & 1.00 & 1.00 & 1.00 & 1.00 \\
\hline 100 & 1.00 & 1.00 & 1.00 & 1.00 & 1.00 & 1.00 & 1.00 & 1.00 & 1.00 \\
\hline 250 & 1.00 & 1.00 & 1.00 & 1.00 & 1.00 & 1.00 & 1.00 & 1.00 & 1.00 \\
\hline $\mathrm{T}$ & \multicolumn{3}{|c|}{ Idiosyncratic Panel $-\rho$} & \multicolumn{3}{|c|}{ Idiosyncratic Group - $\rho$} & \multicolumn{3}{|c|}{ Aznar/Johansen } \\
\hline 50 & 1.00 & 1.00 & 1.00 & 1.00 & 1.00 & 1.00 & 0.90 & 0.90 & 0.91 \\
\hline 100 & 1.00 & 1.00 & 1.00 & 1.00 & 1.00 & 1.00 & 0.97 & 0.96 & 0.97 \\
\hline 250 & 1.00 & 1.00 & 1.00 & 1.00 & 1.00 & 1.00 & 0.98 & 0.99 & 0.99 \\
\hline
\end{tabular}


Table 8: Cointegration in $F_{t}$ but not in $E_{i, t}$ with MA errors.

\begin{tabular}{|c|c|c|c|c|c|c|c|c|c|}
\hline $\mathrm{N}$ & 25 & 50 & 100 & 25 & 50 & 100 & 25 & 50 & 100 \\
\hline $\mathrm{T}$ & \multicolumn{3}{|c|}{ Kao- $\rho^{*}$} & \multicolumn{3}{|c|}{ Kao- $A D F$} & \multicolumn{3}{|c|}{ Pedroni Panel $-\rho$} \\
\hline 50 & 0.18 & 0.17 & 0.24 & 0.55 & 0.60 & 0.65 & 0.01 & 0.00 & 0.01 \\
\hline 100 & 0.25 & 0.29 & 0.39 & 0.59 & 0.69 & 0.73 & 0.07 & 0.03 & 0.11 \\
\hline 250 & 0.37 & 0.40 & 0.50 & 0.70 & 0.77 & 0.78 & 0.18 & 0.10 & 0.32 \\
\hline $\mathrm{T}$ & \multicolumn{3}{|c|}{ Pedroni Panel $-t$} & \multicolumn{3}{|c|}{ Pedroni Group $-\rho$} & \multicolumn{3}{|c|}{ Pedroni Group - $t$} \\
\hline 50 & 0.05 & 0.03 & 0.06 & 0.00 & 0.00 & 0.00 & 0.03 & 0.02 & 0.05 \\
\hline 100 & 0.09 & 0.06 & 0.19 & 0.05 & 0.01 & 0.10 & 0.07 & 0.03 & 0.15 \\
\hline 250 & 0.17 & 0.11 & 0.33 & 0.25 & 0.13 & 0.57 & 0.19 & 0.10 & 0.42 \\
\hline $\mathrm{T}$ & \multicolumn{3}{|c|}{ Idiosyncratic Panel $-\rho$} & \multicolumn{3}{|c|}{ Idiosyncratic Group $-\rho$} & \multicolumn{3}{|c|}{ Aznar/Johansen } \\
\hline 50 & 0.00 & 0.00 & 0.00 & 0.00 & 0.00 & 0.00 & 0.61 & 0.61 & 0.74 \\
\hline 100 & 0.00 & 0.00 & 0.00 & 0.00 & 0.00 & 0.00 & 0.70 & 0.78 & 0.85 \\
\hline 250 & 0.02 & 0.00 & 0.00 & 0.00 & 0.00 & 0.00 & 0.68 & 0.85 & 0.87 \\
\hline
\end{tabular}

\begin{tabular}{l}
\hline$F_{t}^{Y}=\sum_{s=1}^{t} f_{s}^{Y}, \Delta F_{t}^{X}=\Delta F_{t}^{Y}+f_{t}^{X}, E_{i, t}=\sum_{s=1}^{T} e_{i, s}$, \\
where $f_{t}$ and $e_{i, t}$ are MA processes generated as described in Section 5.
\end{tabular}

Rejection frequencies are based on 5\% asymptotic critical values. 
Table 9: No cointegration in $F_{t}$ but cointegration in $E_{i, t}$ with MA errors.

\begin{tabular}{|c|c|c|c|c|c|c|c|c|c|}
\hline $\mathrm{N}$ & 25 & 50 & 100 & 25 & 50 & 100 & 25 & 50 & 100 \\
\hline $\mathrm{T}$ & \multicolumn{3}{|c|}{ Kao- $\rho^{*}$} & \multicolumn{3}{|c|}{ Kao- $A D F$} & \multicolumn{3}{|c|}{ Pedroni Panel $-\rho$} \\
\hline 50 & 0.26 & 0.28 & 0.33 & 0.57 & 0.60 & 0.64 & 0.05 & 0.13 & 0.10 \\
\hline 100 & 0.36 & 0.43 & 0.46 & 0.62 & 0.72 & 0.72 & 0.16 & 0.39 & 0.32 \\
\hline 250 & 0.46 & 0.52 & 0.55 & 0.71 & 0.77 & 0.78 & 0.31 & 0.53 & 0.48 \\
\hline $\mathrm{T}$ & \multicolumn{3}{|c|}{ Pedroni Panel $-t$} & \multicolumn{3}{|c|}{ Pedroni Group $-\rho$} & \multicolumn{3}{|c|}{ Pedroni Group - $t$} \\
\hline 50 & 0.14 & 0.23 & 0.23 & 0.01 & 0.03 & 0.03 & 0.10 & 0.17 & 0.18 \\
\hline 100 & 0.20 & 0.42 & 0.38 & 0.06 & 0.32 & 0.18 & 0.14 & 0.35 & 0.29 \\
\hline 250 & 0.29 & 0.52 & 0.50 & 0.21 & 0.68 & 0.40 & 0.24 & 0.55 & 0.44 \\
\hline $\mathrm{T}$ & \multicolumn{3}{|c|}{ Idiosyncratic Panel $-\rho$} & \multicolumn{3}{|c|}{ Idiosyncratic Group $-\rho$} & \multicolumn{3}{|c|}{ Aznar/Johansen } \\
\hline 50 & 0.92 & 0.99 & 1.00 & 0.94 & 1.00 & 1.00 & 0.12 & 0.12 & 0.11 \\
\hline 100 & 1.00 & 1.00 & 1.00 & 1.00 & 1.00 & 1.00 & 0.10 & 0.11 & 0.10 \\
\hline 250 & 1.00 & 1.00 & 1.00 & 1.00 & 1.00 & 1.00 & 0.09 & 0.10 & 0.08 \\
\hline
\end{tabular}

$F_{t}=\sum_{s=1}^{t} f_{s}, E_{i, t}^{Y}=\sum_{s=1}^{T} e_{i, s}^{Y}, \Delta E_{i, t}^{X}=\Delta E_{i, t}^{Y}+e_{i, s}^{X}$

where $f_{t}$ and $e_{i, t}$ are $M A$ processes generated as described in Section 5.

Rejection frequencies are based on $5 \%$ asymptotic critical values. 
Table 10: Cointegration in $F_{t}$ and $E_{i, t}$ with common cointegrating vector and MA errors.

\begin{tabular}{|c|c|c|c|c|c|c|c|c|c|}
\hline$\overline{\mathrm{N}}$ & 25 & 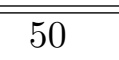 & 100 & 25 & 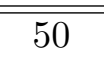 & 100 & 25 & 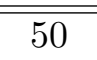 & 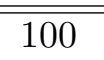 \\
\hline $\mathrm{T}$ & \multicolumn{3}{|c|}{ Kao- $\rho^{*}$} & \multicolumn{3}{|c|}{ Kao- $A D F$} & \multicolumn{3}{|c|}{ Pedroni Panel $-\rho$} \\
\hline 50 & 0.41 & 0.43 & 0.47 & 0.57 & 0.58 & 0.61 & 0.58 & 0.63 & 0.69 \\
\hline 100 & 0.50 & 0.56 & 0.59 & 0.60 & 0.67 & 0.69 & 0.84 & 0.91 & 0.97 \\
\hline 250 & 0.60 & 0.63 & 0.63 & 0.69 & 0.72 & 0.74 & 0.96 & 0.99 & 1.00 \\
\hline $\mathrm{T}$ & \multicolumn{3}{|c|}{ Pedroni Panel $-t$} & \multicolumn{3}{|c|}{ Pedroni Group $-\rho$} & \multicolumn{3}{|c|}{ Pedroni Group $-t$} \\
\hline 50 & 0.69 & 0.77 & 0.84 & 0.74 & 0.81 & 0.91 & 0.86 & 0.94 & 0.98 \\
\hline 100 & 0.87 & 0.94 & 0.98 & 1.00 & 1.00 & 1.00 & 1.00 & 1.00 & 1.00 \\
\hline 250 & 0.95 & 0.98 & 1.00 & 1.00 & 1.00 & 1.00 & 1.00 & 1.00 & 1.00 \\
\hline $\mathrm{T}$ & \multicolumn{3}{|c|}{ Idiosyncratic Panel - $\rho$} & \multicolumn{3}{|c|}{ Idiosyncratic Group $-\rho$} & \multicolumn{3}{|c|}{ Aznar/Johansen } \\
\hline 50 & 0.98 & 1.00 & 1.00 & 0.99 & 1.00 & 1.00 & 0.67 & 0.65 & 0.75 \\
\hline 100 & 1.00 & 1.00 & 1.00 & 1.00 & 1.00 & 1.00 & 0.79 & 0.83 & 0.89 \\
\hline 250 & 1.00 & 1.00 & 1.00 & 1.00 & 1.00 & 1.00 & 0.78 & 0.87 & 0.92 \\
\hline
\end{tabular}

Table 11: Panel no-cointegration tests for observed data

\begin{tabular}{|lccc|}
\hline \hline & Kao $-\rho^{*}$ & Kao - ADF & Pedroni - Panel $-\rho$ \\
Statistic & -4.36 & -3.58 & -2.16 \\
p-value & 0.00 & 0.00 & 0.02 \\
& & & \\
& Pedroni - Panel $-t$ & Pedroni - Group $-\rho$ & Pedroni - Group $-t$ \\
& & & -0.50 \\
Statistic & -1.57 & -0.18 & 0.31 \\
p-value & 0.06 & 0.43 & \\
\hline \hline
\end{tabular}


Table 12: Panel no-cointegration tests for defactored data

\begin{tabular}{|ccc|}
\hline \hline & Statistic & p-value \\
\hline Panel $-\rho$ & -1.58 & 0.57 \\
Panel $-t$ & -1.31 & 0.10 \\
Group $-\rho$ & 0.14 & 0.56 \\
Group $-t$ & -0.05 & 0.48 \\
\hline & Statistic & Critical value \\
Johansen & 8.19 & 12.53 \\
\hline \hline
\end{tabular}

